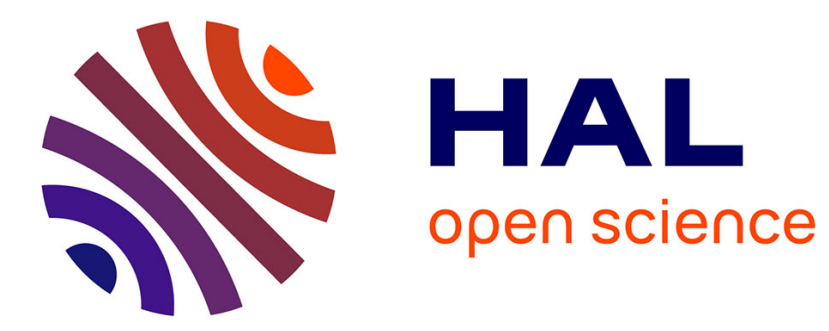

\title{
Parasitism within mutualist guilds explains the maintenance of diversity in multi-species mutualisms
}

Maria Martignoni, Miranda Hart, Jimmy Garnier, Rebecca Tyson

\section{To cite this version:}

Maria Martignoni, Miranda Hart, Jimmy Garnier, Rebecca Tyson. Parasitism within mutualist guilds explains the maintenance of diversity in multi-species mutualisms. Theoretical Ecology, 2020, 10.1007/s12080-020-00472-9 . hal-03006662

\section{HAL Id: hal-03006662 \\ https://hal.science/hal-03006662}

Submitted on 17 Nov 2020

HAL is a multi-disciplinary open access archive for the deposit and dissemination of scientific research documents, whether they are published or not. The documents may come from teaching and research institutions in France or abroad, or from public or private research centers.
L'archive ouverte pluridisciplinaire HAL, est destinée au dépôt et à la diffusion de documents scientifiques de niveau recherche, publiés ou non, émanant des établissements d'enseignement et de recherche français ou étrangers, des laboratoires publics ou privés. 


\title{
Parasitism within mutualist guilds explains the maintenance of diversity in multi-species mutualisms
}

\author{
Maria Martignoni ${ }^{* 1}$, Miranda M. Hart ${ }^{2}$, Jimmy Garnier ${ }^{3}$, and Rebecca C. Tyson ${ }^{4}$ \\ ${ }^{1}$ Department of Mathematics, University of British Columbia, Kelowna (Canada), \\ maria.martignonimseya@ubc.ca \\ ${ }^{2}$ Department of Biology, University of British Columbia, Kelowna (Canada), miranda.hart@ubc.ca \\ ${ }^{3}$ Laboratoire de Mathématiques (LAMA), CNRS and Université de Savoie-Mont Blanc, Chambery \\ (France), jimmy.garnier@univ-smb.fr \\ ${ }^{4}$ Department of Mathematics, University of British Columbia, Kelowna (Canada), \\ rebecca.tyson@ubc.ca
}

November 16, 2020

\begin{abstract}
We consider here mutualisms where there are multiple species sharing a resource supplied by the same partner. If, as commonly assumed, there is competition between the species, then only the superior competitor should persist. Nevertheless, coexistence of multiple species sharing the same mutualistic partner is a widespread phenomenon. Regulation of nutrient exchange, where each species receives resources from the partner in proportion to the strength of the mutualism between the two, has been proposed as the main mechanism for coexistence in multi-species mutualisms involving the transfer of nutrients. Significant arguments, however, challenge the importance of partner selection processes. We present a mathematical model, applied to the arbuscular mycorrhizal symbiosis, to propose an alternative explanation for this coexistence. We show that asymmetric resource exchange between the plant and its fungal guild can lead to indirect parasitic interactions between guild members. In our model, the amount of carbon supplied by the plant to the fungi depends on both plant and fungal biomass, while the amount of phosphorus supplied by the fungi to the plant depends on both plant and fungal biomass when the plant is small, and effectively on fungal biomass only when the plant is large. As a consequence of these functional responses, more beneficial mutualists increase resource availability, and are indirectly exploited by less beneficial species that consume the resource and grow larger than they would in the absence of the better mutualists. As guild mutualists are not competing, competitive exclusion does not occur. Hence, the interaction structure can explain the maintenance of diversity within guilds in the absence of spatial structure and niche-related processes.
\end{abstract}

Keywords : Mutualism, mutualist guilds, coexistence, arbuscular mycorrhizal fungi, indirect interactions, mathematical model, ordinary differential equations, functional response, density-dependent resource exchange.

*corresponding author 


\section{Introduction}

Within mutualist guilds, the principle of competitive exclusion seems not to apply. Indeed, within a guild, mutualists occupying closely related niches coexist on the same partner (Palmer et al., 2003). This fact presents a paradox: According to the principle of competitive exclusion one would expect less beneficial mutualists, i.e., species that provide a lower amount of nutrient in return for the benefit received, to dominate and outcompete more beneficial mutualists (Palmer et al., 2003; Hardin, 1960). Yet, mutualisms involving only two interacting species are relatively rare (Hoeksema and Bruna, 2000; Herre et al., 1999). New evidence has revealed that even mutualisms previously thought to be species-specific, such as for example the fig tree-fig wasp mutualism, are now recognised to involve a guild of mutualists with very similar habits and morphology (Molbo et al., 2003; Pellmyr, 1999; Knowlton and Rohwer, 2003; Herre et al., 1999; Hoeksema and Bruna, 2000).

The arbuscular mycorrhizal (AM) mutualism is another example that is particularly challenging to explain. AM fungi exist in the roots of most terrestrial plants (Smith and Read, 2010). The hyphae of the fungi grow from the plant roots into the soil, where they are able to efficiently increase the absorption of nutrients that are limiting to plant growth (e.g., phosphorus). In return for these nutrients, the host plant provides carbon to the fungi. In nature, a single host plant often associates with dozens of AM fungal species, many of them functionally indistinguishable (Herre et al., 1999; Hoeksema and Bruna, 2000; Öpik et al., 2006). Guild members coexist tightly in space within the roots and in the soil (Bennett and Bever, 2009; Aldrich-Wolfe, 2007), rarely displaying the patchy distribution typically associated with niche differentiation or aggregation (Powell and Bennett, 2016).

There has been some investigation as to the mechanism for this coexistence. Niche differences (Batstone et al., 2018), colonization-competition trade-offs (Smith et al., 2018), spatial structure (Wilson et al., 2003), or physio-evolutionary feedbacks (Bever, 2015), can facilitate coexistence in trophic communities as well as in multi-species mutualisms. None of these mechanisms, however, can explain coexistence of species with a high degree of niche overlap in the absence of spatial structure.

One of the most popular explanations for coexistence of multiple fungal species has been regulation of resource exchange (Palmer et al., 2003). This mechanism requires that the host favours guild members with more beneficial traits, thereby explaining the persistence of good mutualists. Such preferential allocation of resources has been documented in the AM symbiosis (Kiers et al., 2011; Hammer et al., 2011; Bever et al., 2009; Ji and Bever, 2016) and has theoretical support (Bachelot and Lee, 2018; Bever, 2015; Moeller and Neubert, 2016; Hoeksema and Kummel, 2003; Christian and Bever, 2018; Křivan and Revilla, 2019; Valdovinos et al., 2013). However, experimental studies have yielded inconsistent results (Fitter, 2006; Kiers and Van Der Heijden, 2006; Walder and van der Heijden, 2015) and no physiological basis for host discrimination has been identified. In addition, those experiments showing preferential allocation by the plant (allocation of plant carbon to fungi in proportion to the benefit received from each fungus) relied on an artificial spatial structure where the fungi were spatially segregated (Kiers et al., 2011; Bever et al., 2009; Walder et al., 2012).

We propose an alternative mechanism that stabilises coexistence of multiple AM fungi sharing a single host. We develop a mathematical model to show that asymmetric biomassdependent resource exchange can lead to stable coexistence of multiple AM fungal species, without appealing to niche-based mechanisms, spatial segregation, or preferential allocation. In our model, carbon transfer depends on plant and fungal biomass, while phosphorus transfer depends on both plant and fungal biomass when the plant is small, and effectively on fungal biomass only when the plant is large. We show that this asymmetry in nutrient exchange results in indirect parasitism between fungal mutualists, where less mutualistic members indi- 
To investigate the dynamics of multi-species mutualisms we develop a model for the interactions between a host plant and its associated fungal mutualists. Resources exchanged are phosphorus (fungi to plant) and carbon (plant to fungi). Here, the changes in plant and fungal biomass are related to the amount of resource received, to the amount of resource given, and to maintenance costs. All of the mutualists coexist on a single plant, i.e., the carbon provided by the single plant supports the growth of all of the mutualists. The amount of resource exchanged depends on the plant and fungal biomass. We use 'plant biomass' to indicate the individual below- and above-ground parts. As it is not feasible to define fungal biomass at an individual scale, we use the term 'fungal biomass' to refer to the population biomass of a fungal species. In our model therefore, this scenario can be represented by a series of differential equations describing the change over time of plant biomass $(p)$ and of the biomass $\left(m_{i}\right)$ of each of the $N$ species of AM fungi $\left(m_{1}, m_{2}, . ., m_{N}\right)$ :

$$
\begin{aligned}
& \overbrace{\frac{d p}{d t}}^{\begin{array}{c}
\text { change in } \\
\text { plant biomass }
\end{array}}=q_{h p} \overbrace{\sum_{i}\left(\alpha_{i} m_{i}\right) \frac{p}{d+p}}^{\begin{array}{c}
\text { phosphorus } \\
\text { from AM fungi }
\end{array}}-q_{c p} \overbrace{\sum_{i}\left(\beta_{i} m_{i}\right) p}^{\begin{array}{c}
\text { carbon } \\
\text { to AM fungi }
\end{array}}+\overbrace{r_{p} p}^{\begin{array}{c}
\text { intrinsic } \\
\text { growth }
\end{array}}-\overbrace{\mu_{p} p^{n}}^{\begin{array}{c}
\text { maintenance } \\
\text { cost }
\end{array}}, \\
& \underbrace{\frac{d m_{i}}{d t}}_{\begin{array}{c}
\text { change in } \\
\text { fungal biomass }
\end{array}}=q_{c m_{i}} \underbrace{\beta_{i} p m_{i}}_{\begin{array}{c}
\text { carbon } \\
\text { from plant }
\end{array}}-q_{h m_{i}} \underbrace{\alpha_{i} \frac{p}{d+p} m_{i}}_{\begin{array}{c}
\text { phosphorus } \\
\text { to plant }
\end{array}}-\underbrace{\mu_{m_{i}} m_{i}^{s}}_{\begin{array}{c}
\text { maintenance } \\
\text { cost }
\end{array}}, i=1 \ldots N .
\end{aligned}
$$

A brief description of the parameters used in the model is given in Table 1. Here, we present an overview of the main features of the model.

The first terms on the right sides of Eqs. (1a) and (1b) describe the gain in plant and fungal biomass per unit time due to the resource received. The second terms represent the loss in biomass per unit time due to resource given. The relationship between the specific functional forms chosen for these terms is tied to the biology, and discussed in detail in the following section. The last terms for both equations describes the resource required for maintaining the existing biomass. The additional term in Eq. (1a), the intrinsic growth term, represents plant growth per unit time in the absence of the fungi (Smith et al., 2003). AM fungi are obligate mutualists with no saprobic ability and cannot survive in the absence of the host plant, so no intrinsic growth term appears in Eq. (1b).

Fungal species can be differentiated according to four specific traits: the efficiency with which carbon and phosphorus are converted into biomass $\left(q_{c m_{i}}\right.$ and $q_{h m_{i}}$ ) (van Aarle and Olsson, 2003), the ability to provide phosphorus to the plant $\left(\alpha_{i}\right)$ (Ravnskov and Jakobsen, 1995; Drew et al., 2003), the access to host carbon $\left(\beta_{i}\right)$ (Pearson and Jakobsen, 1993; Zhu and Miller, 2003) or the maintenance cost $\left(\mu_{m_{i}}\right)$ (Sylvia and Williams, 1992). Consistent with the literature, fungal species in the model can be distinguished accordingly to these four traits.

The literature provides little evidence about how the maintenance costs of plant and fungi depend on their respective biomasses (last term in Eqs. (1a) and (1b)). As density dependent and non-density dependent population growth are found in nature (Hassell, 1975), we investigate the effect of both linear (i.e. $n=1$ and $s=1$ ) and nonlinear forms of the maintenance cost term (i.e. $n>1$ and $s>1$ ). 


\section{Quantification of resource exchange}

Nutrient exchange between the host plant and the fungal mutualists occurs inside the plant root cells, where the AM fungi build nutrient exchange zones called 'arbuscules' (Peterson and Guinel, 2000; Wang et al., 2017). It is reasonable to assume that the amount of phosphorus transferred increases with increasing root colonization and with increasing hyphal length (Douds et al., 2000; Treseder, 2013; Sawers et al., 2017); More arbuscules means more contact locations where nutrients can be exchanged, and longer hyphae in the soil mean greater fungal access to soil phosphorus. In the model, we thus assume a linear relationship between fungal biomass and phosphorus transfer.

The percentage of root colonization by a fungal species is typically between $20 \%$ and $50 \%$ of plant roots, reaching $80 \%$ at most (Hart and Reader, 2002; Klironomos and Hart, 2002). That is, space availability in the roots seems not to be a factor limiting the presence of AM fungi. Thus, we assume that the plant limits phosphorus transfer only when plant biomass is small, i.e., when less plant availability implies less habitat for the fungus and therefore less phosphorus transfer, but not when the plant is big.

Mathematically, these assumptions lead to the phosphorus transfer term

$$
\text { Phosphorus transfer } \propto m_{i} \frac{p}{d+p} .
$$

where $d$ is the half-saturation constant. When $p \ll d$, phosphorus transfer depends linearly on fungal and plant biomass, while when $p \gg d$, phosphorus transfer depends on fungal biomass only. Eq. (2) corresponds to the second term of Eq. (1a) and to the first term of Eq. (1b). Eq. (2) implies that in the presence of multiple mutualists, each of the mutualists supplies to the plant a quantity of phosphorus per unit time that is proportional to its own biomass. Note that, in our model, the non-linearity in the phosphorus transfer term (Eq. (2)) is due to a limitation that the fungus experiences at low plant biomass, and not a limitation in the benefit of mutualism set by the plant (i.e., the benefit is not saturating at large biomass of the species giving the benefit, as seen in previous models of mutualistic interactions (Holland and DeAngelis, 2010)). Thus, phosphorus transfer is limited by low plant biomass, but is independent of plant biomass (i.e., depends only on fungal biomass) when plant biomass is large.

The literature shows that the plant supplies its associated mutualists with a proportion of the carbon it synthesizes (Douds et al., 2000; Treseder and Cross, 2006; Graham, 2000). It has been shown that carbon transfer from the plant to the AM fungi depends on photosynthetic capacity and on the extent of root colonization by AM fungi (Thomson et al., 1990; Vierheilig et al., 2002). In the model, we assume a linear relationship between plant biomass and carbon fixation. We further assume a linear relationship between carbon fixation and carbon transfer. Finally, we assume linear dependence of carbon transfer and fungal biomass. In sum

$$
\text { Carbon transfer } \propto m_{i} p,
$$

as indicated by the first and second terms of Eqs. (1a) and (1b). The choice of the term for carbon transfer in Eq. (3) implies that when multiple mutualists are associated with the same host, each species is supplied with an amount of host carbon per unit time that its proportional to its individual fungal biomass and to the existing plant biomass. The fungi do not directly compete for carbon, but each accesses a different proportion of host carbon depending on eachfungus' biomass.

One can directly observe that the phosphorus and carbon transfer terms (Eqs. (2) and (3)) show a different dependence on the biomass of the supplying and receiving species. These differences are tied to the biology of the system, as explained above. Resource exchange is 
therefore asymmetric, where carbon transfer depends on both plant and fungal biomass, while phosphorus transfer depends on fungal biomass only, once the plant is large enough.

Although the dependence of the nutrient transfer terms (Eqs. (2) and (3)) on plant and fungal biomass is the same for all fungi, the exact amount of phosphorus and carbon transferred varies with the parameters $\alpha_{i}$ and $\beta_{i}$ respectively, and can vary among species. Fungi characterised by a larger $\alpha_{i}$ and lower $\beta_{i}$ parameters have a higher ability to transfer phosphorus to the plant and take less carbon in return, and therefore can be said to be more mutualistic than species having a smaller $\alpha_{i}$ and larger $\beta_{i}$. Thus, where a fungus lies on the mutualism-parasitism continuum depends on the context provided by the other fungi present (Lekberg and Koide, 2014).

\section{Model analysis and the impact on growth of differences in the resource exchange ability of the fungi}

We will analyse the model of Eq. (1) using both linear stability analysis and numerical simulations. Numerical simulations have been performed using the built-in ode45 solver in MatlabR2017a. First, we will determine the conditions under which a stable mutualism can be established. Second, we will investigate how differences in the resource exchange ability of the fungi affects plant growth and the growth of other guild members. For this purpose, we will study the system dynamics when all model parameters are held at a constant value (given in the caption of Fig. 3) except those that quantify the exchange of nutrients between plant and fungi, i.e., $\alpha_{i}$ and $\beta_{i}$. These parameters represent, respectively, the ability of fungal species $i$ to provide phosphorus to the plant and the access of fungal species $i$ to plant carbon. We will determine analytically how the plant and fungal steady states depend on the resource exchange capacity of the whole guild. We will then validate the analytical results numerically.

Because host discrimination has been one of the most popular explanations for coexistence in multi-species mutualisms (Fitter, 2006; Kiers and Van Der Heijden, 2006; Walder and van der Heijden, 2015), we are interested in seeing how it affects the system dynamics. We will consider the following two cases:

(a) No host discrimination: Access to host carbon is the same for all fungi, regardless of the amount of phosphorus they provide (i.e., parameter $\beta_{i}$ is held constant for all species while $\alpha_{i}$ varies). Since all of the species are characterised by the same carbon transfer rate parameter, cheaters are those that provide little phosphorus (low $\alpha_{i}$ ).

(b) Host discrimination: More beneficial fungi (high phosphorus transfer) have access to more host carbon, while fungi that are less beneficial (low phosphorus transfer), have a reduced access to host carbon (i.e., species with large/low parameter $\alpha_{i}$ also have large $/$ low $\beta_{i}$ ).

\section{Results}

\section{Mutualism establishment and coexistence}

One plant - one fungus mutualism: The establishment of a mutualistic relationship between a plant and one fungal species can occur as long as (i) the plant receives enough nutrients to support its growth (i.e., $\left.\alpha>\left(q_{c p} \beta d\right) / q_{h p}\right)$, (ii) plant and AM fungi are able to make effective use of the resource received (i.e., $\left(q_{c m} q_{h p}\right) /\left(q_{c p} q_{h m}\right)>1$ ), and (iii) maintenance costs are kept low (i.e., $\left.\mu_{p} \mu_{m}<\beta^{2}\left(q_{c m} q_{h p}-q_{h m} q_{c p}\right) /\left(4 q_{h m} q_{h p}\right)\right)$. As long as these three 
conditions are satisfied, the two nullclines in the phase plane of Fig. 1 intersect and coexistence is observed (see SI, section 1 for details).

From the phase plane in Fig. 1 we can also notice that when fungal biomass is large compared to plant biomass, fungal biomass will decrease and plant biomass will increase. The plant will therefore be exploiting the fungi, till its biomass is large enough to allow fungal growth. Similarly, when fungal biomass is low compared to plant biomass, the fungi will not be able to support plant growth. In this case, before showing mutual growth, plant biomass will decrease (suggesting parasitic fungi) and fungal biomass will increase.

Graphical insights into the establishment of mutualistic interactions: Plant growth saturates at the point where there is a balance between the benefit and cost of the mutualism. This balance is reached as a consequence of the asymmetry in phosphorus and carbon transfer (Eqs. (2) and (3)). Fig. 2 demonstrates how the benefits and costs of the mutualism are distributed across the plant and fungus, as a function of plant or fungal biomass. The figure shows plots of the three key functional responses of the mutualism: the gross benefit $(\mathrm{GB})$, cost (C), and net effect (NE), where NE = GB - C. These functions are given in Table 2. In the right panel of Fig. 2, one can see that when plant biomass is small, the gross benefit functional response of the plant is larger than the cost functional response. This situation results in a positive net effect on plant growth, and therefore in an increase in plant biomass. When plant biomass is large, however, the gross benefit functional response decreases, while the cost functional response remains constant, eventually leading to a negative effect on plant growth. Plant biomass is therefore expected to stabilise around a certain value for which the plant gross benefit and cost functional responses are equal to each other. That is, the plant stops growing when the net effect (see Table 2) is zero. Consequently, plant growth is limited by its interaction with the fungal mutualists.

Fungal growth is not limited by its interaction with the host plant. As long as plant biomass is large enough, the net effect of mutualism for the fungi remains positive (see left panel of Fig. 2). Without any kind of self-limitation $(s=1)$ fungal growth is unlimited (see last term in Eq. (1b)). Fungal growth is limited for $s>1$. In either case, the model predicts plantfungi coexistence (see Fig. S6). Plant-fungus coexistence is observed whether the maintenance cost per unit time of the plant (see last term in Eq. (1a)) is linearly proportional to plant biomass $(n=1)$ or increases non-linearly $(n>1)$ with plant biomass (see Fig. S5), and for a positive intrinsic growth rate $r_{p}>0$ (see Fig. S4).

One plant - multiple fungi case: When considering a plant associating with $N$ fungal species, either coexistence or extinction of all mutualists is observed. And as long as (i) at least one of the fungal species provides enough nutrients to the plant (i.e., the inequality $\alpha_{i}>\left(q_{c p} \beta_{i} d\right) / q_{h p}$ is satisfied for at least one species), and as long as (ii) plant and fungi are able to make effective use of the resource received (i.e., $\left(q_{c m} q_{h p}\right) /\left(q_{c p} q_{h m}\right)>1$ ), the mutualism can establish and coexistence is observed. Additionally, for mutualism establishment, it must hold

$$
\text { (iii) } \begin{aligned}
(1+ & Q)^{2}\left(1+\frac{\operatorname{Cov}\left(\boldsymbol{\alpha} / \sqrt{\boldsymbol{\mu}_{m}}, \boldsymbol{\beta} / \sqrt{\boldsymbol{\mu}_{m}}\right)}{\left(\overline{\alpha / \sqrt{\mu_{m}}}\right)\left(\overline{\beta / \sqrt{\mu_{m}}}\right)}\right)^{2} \\
& -4 Q\left(1+\frac{\operatorname{Var}\left(\boldsymbol{\alpha} / \sqrt{\boldsymbol{\mu}_{m}}\right)}{\left(\overline{\alpha / \sqrt{\mu_{m}}}\right)^{2}}\right)\left(1+\frac{\mu_{p}}{N q_{c m} q_{c p}\left(\overline{\beta / \sqrt{\mu_{m}}}\right)^{2}}+\frac{\operatorname{Var}\left(\boldsymbol{\beta} / \sqrt{\boldsymbol{\mu}_{m}}\right)}{\left(\overline{\beta / \sqrt{\mu_{m}}}\right)^{2}}\right)>0,
\end{aligned}
$$

where $\boldsymbol{\alpha}=\left(\alpha_{1}, \ldots, \alpha_{N}\right)$ and $\boldsymbol{\beta}=\left(\beta_{1}, \ldots, \beta_{N}\right)$ represent respectively the ability of the whole guild to transfer phosphorus and get carbon from the plant, $\boldsymbol{\mu}_{m}=\left(\mu_{1}, \ldots, \mu_{N}\right)$ represents 
the maintenance cost of the guild, $\operatorname{Cov}($.$) is the covariance, \operatorname{Var}($.$) is the variance, and \overline{\boldsymbol{\alpha} / \boldsymbol{\mu}_{m}}$ and $\overline{\boldsymbol{\beta} / \boldsymbol{\mu}_{m}}$ represent the mean of the individual resource exchange capacities divided by the maintenance cost of each of the fungi present. Condition (iii) is more likely to be satisfied when the covariance between $\boldsymbol{\alpha}$ and $\boldsymbol{\beta}$ and the mean $\overline{\boldsymbol{\alpha}}$ are large, and when the variances $\operatorname{Var}(\boldsymbol{\alpha})$ and $\operatorname{Var}(\boldsymbol{\beta})$ are small. Additionally, the condition is less likely to be satisfied when the maintenance cost $\boldsymbol{\mu}_{m}$ of the fungi is large (see SI, section 2 for details). Altogether, conditions (i)-(iii) tell us that it is the overall exchange capacity of all mutualists that determines whether coexistence or extinction of the whole guild will be observed. Plant association with a sufficiently beneficial mutualist can compensate for the presence of a minimally beneficial species, or even for a species that does not provide any nutrients to the plant (i.e. $\alpha_{i}=0$ ), as long as the plant receives on average enough nutrients to support its growth, and as long as guild members are similar enough in their ability to transfer phosphorus and get host carbon.

Fig. 3 shows typical results of simulation experiments where the carbon supplied by a single plant is shared among fungi characterized by different resource exchange capacities. In the first two subfigures we investigate the two cases described in the methods: No host discrimination (Fig. 3a) and host discrimination (Fig. 3b). As expected from the model analysis, coexistence is observed whether or not the plant rewards more mutualistic fungi with more carbon. The growth dynamics, however, differ. When all fungi have access to the same amount of carbon independent of the phosphorus supplied, less mutualistic fungi reach a higher abundance than more mutualistic ones (Fig. 3a). Mutualists supplying more phosphorus and accessing more carbon reach a higher biomass than less mutualistic fungi (Fig. 3b). The presence of more mutualistic fungi increases plant biomass, and therefore resource availability. If the plant is supported by enough high quality mutualists, then less mutualistic species can exploit the plant's resource and, indirectly, the more beneficial species. If the guild does not provide enough phosphorus to the host plant (i.e., the conditions for coexistence mentioned above are not satisfied), extinction of the whole guild is observed (see Fig. 3c).

\section{Indirect parasitism among guild members}

Fig. 4 compares plant and fungal growth when the plant is associated with a more mutualistic fungus (species 1), a less mutualistic fungus (species 2), or a combination of the two species. When a less mutualistic fungus (species 2) is considered in pairwise association with the plant, the growth rates and final sizes of the plant and the fungus are lowest. When the plant is considered in pairwise association with the more mutualistic fungus (species 1), growth rates and final sizes are highest. If species 1 and 2 are both present, the growth rate and final size of the plant and species 1 are lowered, while the growth rate of species 2 is increased. The presence of species 2 negatively affects the growth of species 1 , while the presence of species 1 positively affects the growth of species 2 . Indirect interactions between species 1 and 2 are therefore parasitic, with the less mutualistic fungus (species 2) exploiting the more mutualistic one (species 1). The larger the difference in net benefit provided by guild members, the stronger the indirect parasitic interactions. The analysis presented in the SI (section 3) shows that our numerical findings hold for any value of the ability of the fungi to transfer phosphorus (parameter $\alpha_{i}$ ).

These indirect relationships are shown schematically in Fig. 5. The diagram qualitatively represents the interactions of a plant and three fungal species differing in their resource exchange capacities. Species 1 is assumed to be a better mutualist than species 2 and 3, and species 2 is a better mutualist than species 3 . Pairwise direct interactions of the plant with each of the three AM fungal species are mutualistic, where the plant provides equal access to the resource to all three fungi, and receives resource from each species according to its resource exchange ability. Consequently, pairwise indirect interactions among fungal species are 
parasitic, since the less mutualistic species takes advantage of the plant growth resulting from the benefit provided by the more mutualistic species. In other words, species 1 is exploited by 2 and 3 , and species 2 is exploited by 3 .

The model predicts none of the interactions between the fungi to be competitive, i.e., disadvantageous for both parties. In the absence of competitive interactions, competitive exclusion is not expected to occur. Instead, in the pairwise indirect interactions between fungal mutualists, one of the two always benefits. In terms of Fig. 5, each parallel set of arrows between fungi represents interactions that are parasitic, i.e. positive for the less mutualistic species and negative for the more mutualistic one.

\section{Discussion}

\section{Modelling of mutualistic interactions}

I present a model that allows for stable coexistence of multiple multualists sharing a resource supplied by a single partner, in the absence of niche-based mechanisms, spatial structure, or preferential allocation. In the model, multi-species mutualism establishment depends on the overall net benefit provided by the guild to the partner. As long as the plant receives enough nutrients to support its growth, coexistence of all mutualists is observed. Similarly, in public goods games, coexistence of more and less beneficial mutualists has been shown to occur as long as the there are enough good mutualists to produce the public good (Archetti and Scheuring, 2011, 2013). In our model, we obtain the additional requirement that establishment is more likely if the variance across guild members is small, i.e., that the guild members are similar in mutualistic quality.

Previous ordinary differential equation models of mutualistic interactions have shown that stable coexistence in mutualisms involving only two interacting species can be obtained by setting an upper limit to the benefit that each species can provide to the other (i.e., the gross benefit (GB) and cost (C) curves in Fig. 2 level off for sufficiently large biomass) (Wright, 1989; Ingvarsson and Lundberg, 1995; Kot, 2001; May, 1976; Addicott, 1981; Holland et al., 2002; Holland and DeAngelis, 2006; Holland et al., 2004; Holland and DeAngelis, 2009, 2010). However, saturation in the benefit makes stable coexistence of multiple mutualists impossible, in the absence of secondary mechanisms. Inevitably, one of the mutualists will be better than the others at obtaining resource from the partner species. Consequently, the biomass of this species will increase, leading to the consumption of an increasing proportion of the available resource, eventually driving the other mutualists to extinction. On the other hand, symmetric non-saturating resource exchange (corresponding to linear gross benefit (GB) functional responses) results in unlimited growth of both, the mutualists and the partner species (Gause and Witt, 1935; Vandermeer and Boucher, 1978). In our model, the benefit increases linearly with the biomass of the species providing the benefit, and does not saturate at high biomass of the species giving the benefit. Hence, coexistence is possible. At the same time, asymmetry in the benefit and cost of mutualism (Eqs. (2) and (3)) sets a limit to plant growth, which is reached when, for the plant, the benefit and cost of the mutualism equalize.

\section{Mutualism-parasitism continuum}

Experimental work has shown that symbiotic associations are not always necessarily beneficial, but can, under certain circumstances, become parasitic or commensalistic. For example, the nature of the symbiosis may depend on the genotypes of the interacting species or on environmental conditions (Johnson et al., 1997; Lekberg and Koide, 2014). Here, we present 
a mechanism that causes interactions to change from mutualistic to parasitic when there is a disproportion in plant and fungal biomass, where the more abundant species is exploited by the less abundant one before mutual growth can be observed. It has already been hypothesized that depletion in plant growth may be observed when the carbon cost set by the fungi is not sufficiently compensated by phosphorus transfer (Lekberg and Koide, 2014; Smith and Smith, 2012). This mechanism could explain a depletion in plant growth when abundance of AM fungi is low, as observed by Smith and Smith (2012).

\section{Indirect parasitism between guild members}

In this article, we show that the main form of interaction between guild members is indirect parasitism, and as guild members are not competing, competitive exclusion is not expected to occur. The presence of a more mutualistic species increases plant biomass, and therefore resource availability, for other guild members. Each fungal mutualist exploits all of the other species that are more mutualistic fungi than itself, and at the same time is itself exploited by each of the species that are less mutualistic. The most mutualistic species is exploited by all other species, and the least mutualistic one is not exploited by any. The more similar the net benefit provided by the guild members, the more negligible the indirect parasitism and the effect that the mutualist species have on each other's biomass.

Previous work has proposed that stable coexistence of multiple species sharing a limiting resource can emerge as a consequence of indirect interactions in multitrophic communities (Stanton, 2003; McCann, 2000). The presence of a third species may affect the population size of a superior competitor through an interaction chain, e.g., by predation (May, 1972; Caswell, 1978; Allesina and Levine, 2011; Kerr et al., 2002), or it can alter the effect that one species has on another through higher-order interactions, e.g., by varying the amount of resource available (Schoener, 1974; Abrams, 1983; Mayfield and Stouffer, 2017; Levine et al., 2017). In the model presented here, the amount of shared resource available (i.e., plant biomass) is determined by its interaction with the associated mutualists. The fungal species are not directly competing for the resource they are sharing, because the amount of resource available increases with increasing fungal abundance.

\section{Experimental work}

While there is a great deal of work looking at fungal diversity in the microbiome of plants (Husband et al., 2002; Sugiyama et al., 2014) the results are largely focussed on diversity, rather than fungal biomass over time. Only a few studies show the progression of fungal biomass and plant biomass over time when two or more fungal species are present (for example producing data like that shown in plots in Fig. 4).

Hart et al. (2013) found that the abundance of a less mutualistic fungal species is increased by the addition of a better mutualist colonizing the same host plant, and Argüello et al. (2016) showed that a plant receives more phosphorus from a less mutualistic species in the presence of a better mutualist. Jansa et al. (2008) showed that plant and fungal growth in the presence of multiple mutualists were mostly within the range of what was observed when the plant was grown separately with the most and less mutualistic species. All of these results are in agreement with model predictions.

Other observations, however, show that plant growth is higher in the presence of multiple species of AM fungi than in the presence of any of the species separately (Jansa et al., 2008; Alkan et al., 2006; Maherali and Klironomos, 2007; Van der Heijden et al., 1998; Gianinazzi et al., 2010). This fact presents a contradiction with model predictions, as plant biomass is expected to be maximized by the presence of a single, most beneficial species (e.g., species 1 
in Fig. 4). The contraddiction could be explained by the fact that in the model we assume phosphorus supply to be the only benefit provided to the plant by the fungi. Root colonization with AM fungi has also been shown to increase plant resistance to diseases (Jung et al., 2012), protection against parasites (Parniske, 2008), and plant tolerance to abiotic stress (Latef et al., 2016). The presence of fungal species providing complementary benefits can therefore help to maximize plant growth (Afkhami et al., 2014). Further monitoring of plant and fungal growth over time in monocultures of AM fungi and in cultures containing different fungal species is needed for experimental verification of the hypotheses presented here.

\section{Future directions}

Asymmetric nutrient exchange is an important part of the dynamics observed in the model. The veracity of this assumption remains to be tested; very few studies have attempted to bridge theory and empiricism by experimentally testing different mathematical forms of benefit and cost functional responses of mutualisms (Morris et al., 2010; Morales, 2011; Kang et al., 2011; Geib and Galen, 2012). Further empirical work is needed to determine whether mutualistic interactions can effectively be characterised by the asymmetric resource exchange rates assumed in the model.

Additionally, while much can be learned from the model presented here, there are certain features of the system that may be important to include in future modelling efforts. In particular, we do not distinguish between plant structure above or below ground, although plants are known to differentially allocate carbon depending on plant size, environmental conditions or AM fungal abundance (Andersen and Rygiewicz, 1991; Rygiewicz and Andersen, 1994). Also, AM fungi have been shown to contribute to nutrient uptake only after having built a fungal network inside the plant root cells (Dickson et al., 1999). There should therefore exist a threshold of fungal biomass below which the fungi do not contribute to plant growth. Future models may regard plant and fungal biomass as a system of multiple coupled differential equations, to distinguish plant biomass above- and below- ground and intra- and extraradical fungal biomass.

In our work, we also do not consider plant-soil feedback (Van der Putten et al., 2013; Miki, 2012). These types of feedback can indeed influence coexistence patterns (Johnson and Bronstein, 2019). A simple study of the dynamics of these feedbacks could be carried out with the model presented here by considering variations in the intrinsic growth rate of the plant (characterised by parameters $r_{p}$ and $\mu_{p}$ ), as a function of different soils. Our model could also be used as a building block in larger community models which are at present conceptual (Van der Putten et al., 2013) or based on very simple mass action kinetics (Miki, 2012).

Additionally, our model could be used to investigate how different plant-mutualist guild combinations can be more or less evolutionary successful, where the total mutualist quality of the guild depends on the resource exchange capacities of each mutualist in the guild. Such a study would therefore offer an opportunity to couple the physiological qualities of the fungal species present (i.e., their resource exchange capacities) with evolutionary processes (Lekberg and Koide, 2014).

It is important to note that the model presented here is not spatially explicit, and allows for an indefinite number of mutualists to be associated with the same host plant. Space should become a limiting factor, however, at large fungal density (Hepper et al., 1988; Mummey et al., 2009; Koch et al., 2011; Engelmoer et al., 2014). An extension of this model to explicitly considering competition between fungal species is studied by Martignoni et al. (2020). 


\section{${ }_{381}$ Acknowledgment}

382 MMH acknowledges NSERC Discovery Grant RGPIN-2018-237774. JG acknowledges NON-

383 LOCAL project (ANR-14-CE25-0013), GLOBNETS project (ANR-16- CE02-0009) and the

384 European Research Council (ERC) under the European Unions Horizon 2020 research and

385 innovation program (grant agreement No 639638, MesoProbio). RCT acknowledges NSERC

386 Discovery Grant RGPIN-2016-05277 and the 'Make our planet great again (MOPGA)' grant. 
Table 1: Brief description of the parameters of the model of Eq. (1).

\begin{tabular}{|l|l|}
\hline Parameter & Description \\
\hline$\alpha_{i}$ & Phosphorus exchange ability of fungal species $i$ \\
$\beta_{i}$ & Access to host carbon of fungal species $i$ \\
$\mu_{p}$ & Maintenance rate of the plant \\
$\mu_{m_{i}}$ & Maintenance rate of fungal species $i$ \\
$q_{h p}$ & Conversion factor of phosphorus into plant biomass \\
$q_{c p}$ & Conversion factor of carbon into plant biomass \\
$q_{h m_{i}}$ & Conversion factor of phosphorus into fungal biomass of species $i$ \\
$q_{c m_{i}}$ & Conversion factor of carbon into fungal biomass of species $i$ \\
$r_{p}$ & Intrinsic growth rate of the plant \\
$d$ & Half-saturation constant \\
$n$ & Biomass dependence of the plant maintenance rate \\
$s$ & Biomass dependence of the fungal maintenance rate \\
\hline
\end{tabular}

\section{${ }_{387}$ Tables and Figures}

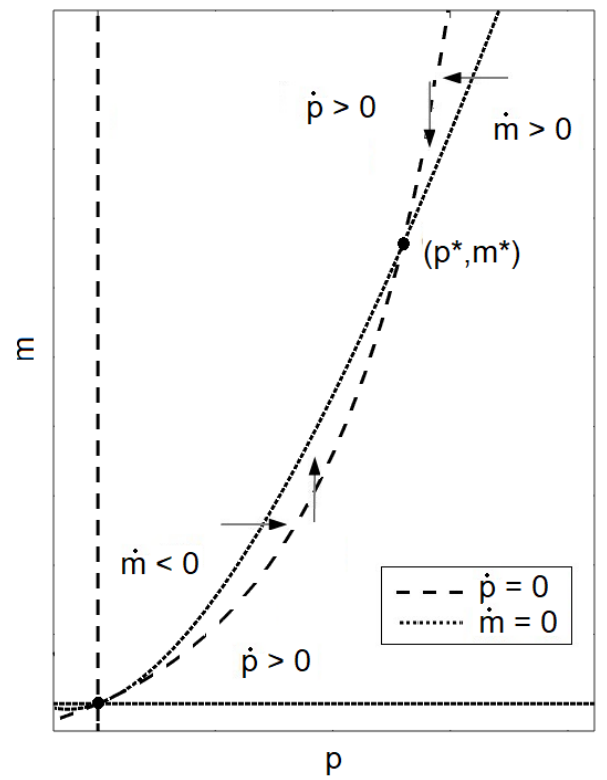

Fig. 1: Nullclines $\dot{p}=0$ (dashed line) and $\dot{p}=0$ (dotted line) of the system of differential equations given in (1) for $N=1$. Arrows represent qualitatively the direction of the flow nearby the stable steady state $\left(p^{*}, m^{*}\right)$. 
Table 2: Biomass-dependence of the gross benefit (GB), cost (C) and net effect (NE) functional responses of plant $(p)$ and a species of AM fungi $\left(m_{i}\right)$, characterizing the model described in Eq. (1). The GB and $\mathrm{C}$ functional responses of plant and fungi are obtained by dividing Eqs. (2) and (3), representing the biomass-dependence of phosphorus/carbon transfer per unit time, by the biomass of the species receiving (GB) or providing (C) the nutrient. The net effect functional response is calculated as the difference between the gross benefit and cost functional responses $(\mathrm{NE}=\mathrm{GB}-\mathrm{C})$.

\begin{tabular}{|l|c|c|l|}
\cline { 2 - 4 } \multicolumn{1}{c|}{} & GB & C & NE \\
\hline Plant (p) & $\frac{m_{i}}{d+p}$ & $m_{i}$ & $m_{i}\left(\frac{1}{d+p}-1\right)$ \\
\hline AM fungi $\left(m_{i}\right)$ & $p$ & $\frac{p}{d+p}$ & $p\left(1-\frac{1}{d+p}\right)$ \\
\hline
\end{tabular}
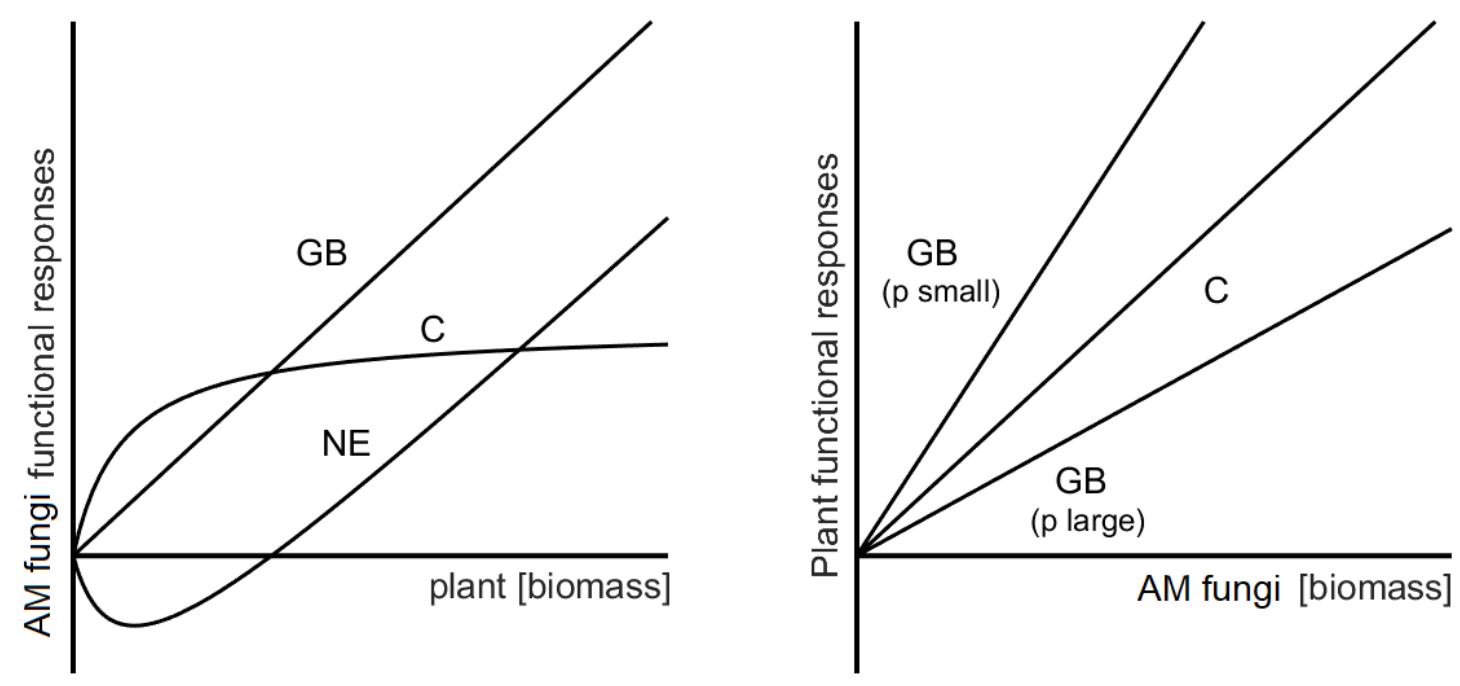

Fig. 2: Qualitative graphical representation of the functional responses of plant and AM fungi given in Table 2. Functional response curves of gross benefit (GB), cost $(\mathrm{C})$ and net effect (NE $=\mathrm{GB}-\mathrm{C}$ ) of a species of AM fungi (left panel) and plant (right panel) are plotted as a function of plant, respectively fungal, biomass. Note that the GB functional response of the plant depends on both, fungal and plant biomass, making the NE functional response of the plant negative at large plant biomass and positive when plant biomass is small. Plant growth will therefore stabilise about a certain value of $p$ for which plant GB and C functional responses are equal to each other. 


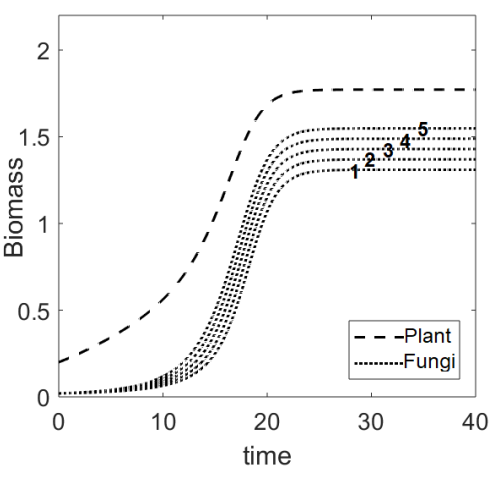

(a)

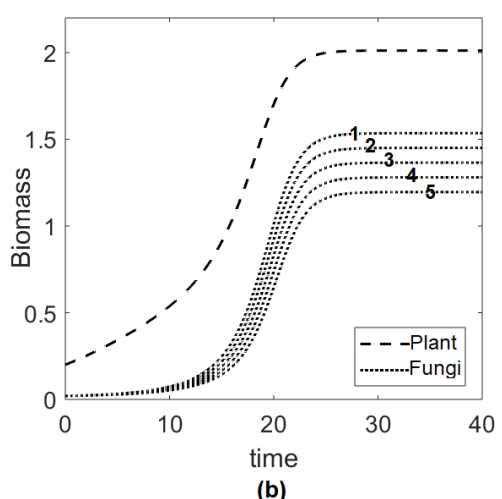

(b)

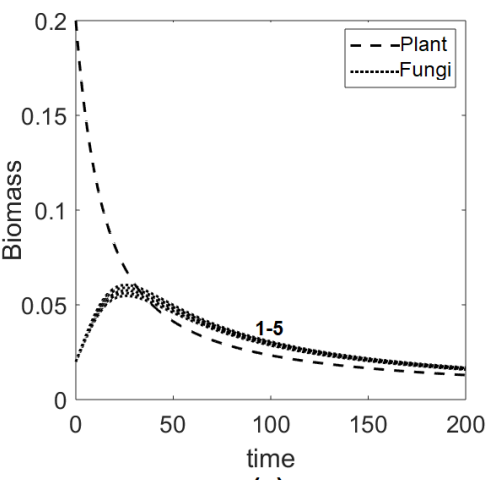

(c)

Fig. 3: Plant and fungal growth over time where (a) fungi differ only in their phosphorus supply to the plant and access to host carbon is the same for all species, (b) fungi supplying the plant with a large/small amount of phosphorus $\left(\alpha_{i}\right)$ have high/low access to host carbon $\left(\beta_{i}\right)$, and (c) fungi provide a very low amount of phosphorus to the plant. Species 1 provides the greatest net benefit to the plant, and species 5 provides the lowest. The plant $p$ is represented as a dashed line, while different fungal species $m_{i}$ are dotted lines, with the numbers close to each curve indicating the species number. Numerical solutions are obtained by solving Eq. (1) for $i=1$.. 5 where (a) $\alpha_{i}$ decreases from 0.62 to 0.38 in steps of size 0.08 , while $\beta_{i}=0.4$ for all species. (b) $\alpha_{i}$ increases from 0.40 to 0.48 in steps of size 0.02 , for $i=1 . .5$ and $\beta_{i}$ decreases from 0.38 to 0.30 in steps of size 0.02 . (c) $\alpha_{i}$ decreases from 0.2 to 0.12 in steps of size 0.02 , while $\beta_{i}=0.4$ for all species. Other parameters are $q_{h p}=3$, $q_{c m_{i}}=2, q_{c p}=q_{h m_{i}}=1, r_{p}=0.04, \mu_{p}=0.3, \mu_{m}=0.8, n=s=2$ and $d=1.2$, for all species. Initial abundances are $p(0)=0.2, m_{i}(0)=0.02$. 

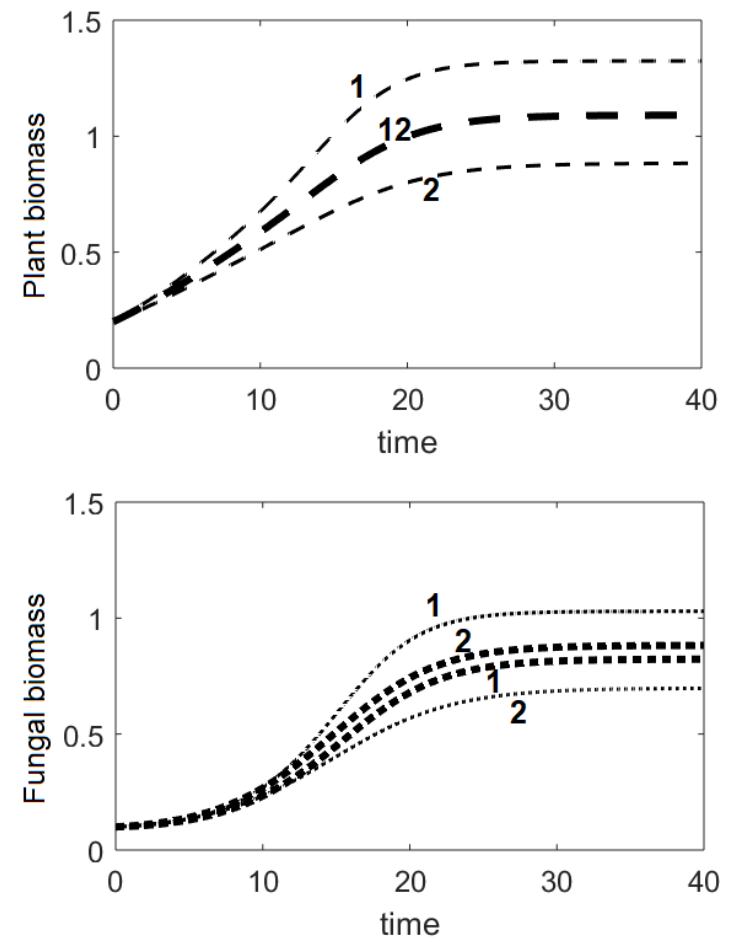

(a)
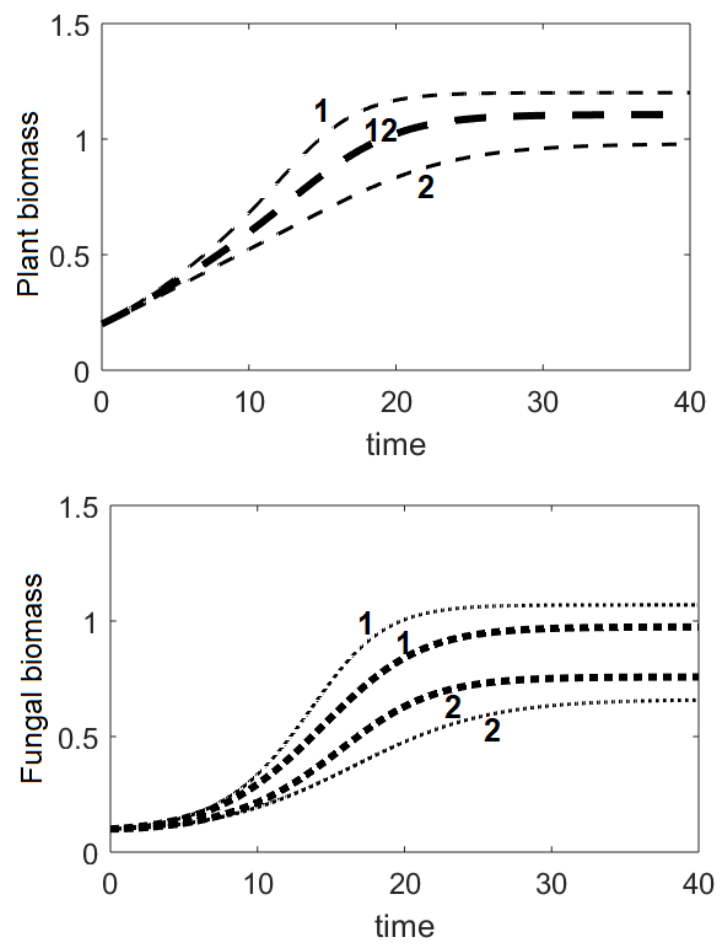

(b)

Fig. 4: Growth over time of a single plant associating with two species of AM fungi. Species 1 is more mutualistic and provides the plant with more phosphorus than species $2\left(\alpha_{1}>\alpha_{2}\right)$. (a) Access to host carbon is the same for both species $\left(\beta_{1}=\beta_{2}\right)$ or (b) Species 1 have access to more host carbon than species $2\left(\beta_{1}>\beta_{2}\right)$. In both cases, the upper figures represent plant biomass over time when the mycorrhizal association is between a plant and two individuals of the more mutualistic species (thin dashed curves, number 1), of the less mutualistic species (thin dashed curves, number 2) or a combination of species 1 and species 2 (thick dashed curves, 12). The bottom figures represent fungal biomass over time when the fungal species are considered in separate association with the plant (thin dotted curves) or grown simultaneously on the same plant (thick dotted curves). Parameters are: $\alpha_{1}=0.45$ and $\alpha_{2}=0.35$, (a) $\beta_{1}=0.45$ and $\beta_{2}=0.35$, (b) $\beta_{1}=\beta_{2}=0.4$. Other parameters correspond to those for Fig. 3. Note that we are using parameter values for which plant growth is always enhanced in the presence of AM fungi. 


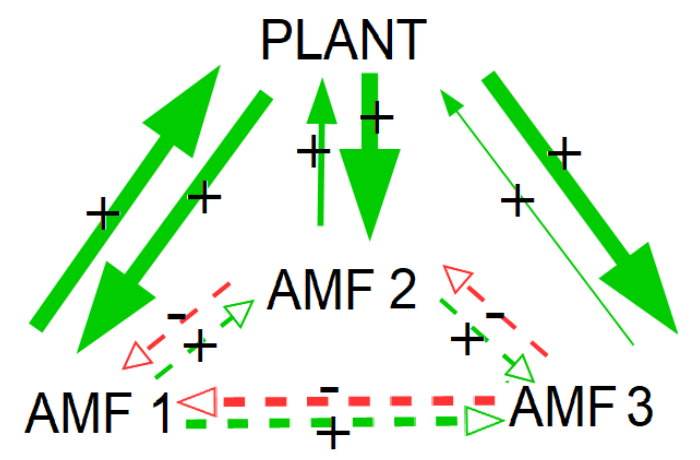

Fig. 5: Diagram representation of direct (solid arrows) and indirect (dotted arrows) interactions between a plant and its associated mutualist guild. Species 1 is more mutualistic than species 2 , and species 2 is more mutualistic than species 3. Arrows indicate the direction of each interaction, with the sender at the tail of the arrow and the receiver at the head. Green arrows represent positive effects $\left({ }^{\prime}+'\right)$, while red arrows represent negative effects on growth ('-'). Arrows thickness qualitatively represents the interaction strength. A '+'/'-' indicates that the sender has a positive/negative effect on the growth of the receiver. A mutualistic interaction is one in which the two parallel interactions are denoted as ('+', ' + '), while a parasitic interaction is one in which one of the parallel interactions is positive and the other is negative $\left({ }^{\prime}+{ }^{\prime},{ }^{\prime}-'\right)$. A competitive interaction is one in which each species negatively impacts the other one, and so it would be labeled $\left({ }^{\prime}-{ }^{\prime},{ }^{\prime}\right.$ '). Interactions between guild members are not competitive $\left({ }^{\prime}-{ }^{\prime},{ }^{\prime}-'\right)$, but parasitic $\left({ }^{\prime}+,{ }^{\prime},{ }^{\prime}\right)$.

\section{References}

Abrams, P. A. (1983). Arguments in favor of higher order interactions. The American Naturalist, 121(6):887-891.

Addicott, J. F. (1981). Stability properties of 2-species models of mutualism: simulation studies. Oecologia, 49(1):42-49.

Afkhami, M. E., Rudgers, J. A., and Stachowicz, J. J. (2014). Multiple mutualist effects: conflict and synergy in multispecies mutualisms. Ecology, 95(4):833-844.

Aldrich-Wolfe, L. (2007). Distinct mycorrhizal communities on new and established hosts in a transitional tropical plant community. Ecology, 88(3):559-566.

Alkan, N., Gadkar, V., Yarden, O., and Kapulnik, Y. (2006). Analysis of quantitative interactions between two species of arbuscular mycorrhizal fungi, glomus mosseae and $\mathrm{g}$. intraradices, by real-time pcr. Applied and Environmental Microbiology, 72(6):4192-4199.

Allesina, S. and Levine, J. M. (2011). A competitive network theory of species diversity. Proceedings of the National Academy of Sciences.

Andersen, C. P. and Rygiewicz, P. T. (1991). Stress interactions and mycorrhizal plant response: understanding carbon allocation priorities. Environmental Pollution, 73(3-4):217244.

Archetti, M. and Scheuring, I. (2011). Coexistence of cooperation and defection in public goods games. Evolution: International Journal of Organic Evolution, 65(4):1140-1148.

Archetti, M. and Scheuring, I. (2013). Trading public goods stabilizes interspecific mutualism. Journal of theoretical biology, 318:58-67. 
Argüello, A., O'Brien, M. J., van der Heijden, M. G., Wiemken, A., Schmid, B., and Niklaus, P. A. (2016). Options of partners improve carbon for phosphorus trade in the arbuscular mycorrhizal mutualism. Ecology Letters, 19(6):648-656.

Bachelot, B. and Lee, C. T. (2018). Dynamic preferential allocation to arbuscular mycorrhizal fungi explains fungal succession and coexistence. Ecology, 99(2):372-384.

Batstone, R. T., Carscadden, K. A., Afkhami, M. E., and Frederickson, M. E. (2018). Using niche breadth theory to explain generalization in mutualisms. Ecology, 99(5):1039-1050.

Bennett, A. E. and Bever, J. D. (2009). Trade-offs between arbuscular mycorrhizal fungal competitive ability and host growth promotion in plantago lanceolata. Oecologia, 160(4):807816.

Bever, J. D. (2015). Preferential allocation, physio-evolutionary feedbacks, and the stability and environmental patterns of mutualism between plants and their root symbionts. New Phytologist, 205(4):1503-1514.

Bever, J. D., Richardson, S. C., Lawrence, B. M., Holmes, J., and Watson, M. (2009). Preferential allocation to beneficial symbiont with spatial structure maintains mycorrhizal mutualism. Ecology letters, 12(1):13-21.

Caswell, H. (1978). Predator-mediated coexistence: a nonequilibrium model. The American Naturalist, 112(983):127-154.

Christian, N. and Bever, J. D. (2018). Carbon allocation and competition maintain variation in plant root mutualisms. Ecology and Evolution.

Dickson, S., Smith, S., and Smith, F. (1999). Characterization of two arbuscular mycorrhizal fungi in symbiosis with allium porrum: colonization, plant growth and phosphate uptake. The New Phytologist, 144(1):163-172.

Douds, D. D., Pfeffer, P. E., and Shachar-Hill, Y. (2000). Carbon partitioning, cost, and metabolism of arbuscular mycorrhizas. In Arbuscular mycorrhizas: physiology and function, pages 107-129. Springer.

Drew, E., Murray, R., Smith, S., and Jakobsen, I. (2003). Beyond the rhizosphere: growth and function of arbuscular mycorrhizal external hyphae in sands of varying pore sizes. Plant and Soil, 251(1):105-114.

Engelmoer, D. J., Behm, J. E., and Toby Kiers, E. (2014). Intense competition between arbuscular mycorrhizal mutualists in an in vitro root microbiome negatively affects total fungal abundance. Molecular ecology, 23(6):1584-1593.

Fitter, A. (2006). What is the link between carbon and phosphorus fluxes in arbuscular mycorrhizas? a null hypothesis for symbiotic function. New Phytologist, 172(1):3-6.

Gause, G. and Witt, A. (1935). Behavior of mixed populations and the problem of natural selection. The American Naturalist, 69(725):596-609.

Geib, J. C. and Galen, C. (2012). Tracing impacts of partner abundance in facultative pollination mutualisms: from individuals to populations. Ecology, 93(7):1581-1592. 
Gianinazzi, S., Gollotte, A., Binet, M.-N., van Tuinen, D., Redecker, D., and Wipf, D. (2010). Agroecology: the key role of arbuscular mycorrhizas in ecosystem services. Mycorrhiza, 20(8):519-530.

Graham, J. (2000). assessing costs of arbuscular mycorrhizal symbiosis agroecosystems fungi. in current advances in mycorrhizae research.

Hammer, E. C., Pallon, J., Wallander, H., and Olsson, P. A. (2011). Tit for tat? a mycorrhizal fungus accumulates phosphorus under low plant carbon availability. FEMS Microbiology Ecology, 76(2):236-244.

Hardin, G. (1960). The competitive exclusion principle. science, 131(3409):1292-1297.

Hart, M. M., Forsythe, J., Oshowski, B., Bücking, H., Jansa, J., and Kiers, E. T. (2013). Hiding in a crowd - does diversity facilitate persistence of a low-quality fungal partner in the mycorrhizal symbiosis? Symbiosis, 59(1):47-56.

Hart, M. M. and Reader, R. J. (2002). Taxonomic basis for variation in the colonization strategy of arbuscular mycorrhizal fungi. New Phytologist, 153(2):335-344.

Hassell, M. (1975). Density-dependence in single-species populations. The Journal of animal ecology, pages 283-295.

Hepper, C. M., Azcon-Aguilar, C., Rosendahl, S., and Sen, R. (1988). Competition between three species of glomus used as spatially separated introduced and indigenous mycorrhizal inocula for leek (allium porrum 1.). New Phytologist, 110(2):207-215.

Herre, E. A., Knowlton, N., Mueller, U. G., and Rehner, S. A. (1999). The evolution of mutualisms: exploring the paths between conflict and cooperation. Trends in Ecology 8 Evolution, 14(2):49-53.

Hoeksema, J. D. and Bruna, E. M. (2000). Pursuing the big questions about interspecific mutualism: a review of theoretical approaches. Oecologia, 125(3):321-330.

Hoeksema, J. D. and Kummel, M. (2003). Ecological persistence of the plant-mycorrhizal mutualism: a hypothesis from species coexistence theory. the american naturalist, 162(S4):S40S50.

Holland, J. and DeAngelis, D. (2006). Interspecific population regulation and the stability of mutualism: fruit abortion and density-dependent mortality of pollinating seed-eating insects. Oikos, 113(3):563-571.

Holland, J. N. and DeAngelis, D. L. (2009). Consumer-resource theory predicts dynamic transitions between outcomes of interspecific interactions. Ecology Letters, 12(12):13571366.

Holland, J. N. and DeAngelis, D. L. (2010). A consumer-resource approach to the densitydependent population dynamics of mutualism. Ecology, 91(5):1286-1295.

Holland, J. N., DeAngelis, D. L., and Bronstein, J. L. (2002). Population dynamics and mutualism: functional responses of benefits and costs. The American Naturalist, 159(3):231-244.

Holland, J. N., DeAngelis, D. L., and Schultz, S. T. (2004). Evolutionary stability of mutualism: interspecific population regulation as an evolutionarily stable strategy. Proceedings of the Royal Society of London B: Biological Sciences, 271(1550):1807-1814. 
Husband, R., Herre, E. A., Turner, S., Gallery, R., and Young, J. (2002). Molecular diversity of arbuscular mycorrhizal fungi and patterns of host association over time and space in a tropical forest. Molecular Ecology, 11(12):2669-2678.

Ingvarsson, P. K. and Lundberg, S. (1995). Pollinator functional response and plant population dynamics: pollinators as a limiting resource. Evolutionary Ecology, 9(4):421-428.

Jansa, J., Smith, F. A., and Smith, S. E. (2008). Are there benefits of simultaneous root colonization by different arbuscular mycorrhizal fungi? New Phytologist, 177(3):779-789.

Ji, B. and Bever, J. D. (2016). Plant preferential allocation and fungal reward decline with soil phosphorus: implications for mycorrhizal mutualism. Ecosphere, 7(5):e01256.

Johnson, C. A. and Bronstein, J. L. (2019). Coexistence and competitive exclusion in mutualism. Ecology, 100(6):e02708.

Johnson, N. C., Graham, J.-H., and Smith, F. A. (1997). Functioning of mycorrhizal associations along the mutualism-parasitism continuum. New phytologist, 135(4):575-585.

Jung, S. C., Martinez-Medina, A., Lopez-Raez, J. A., and Pozo, M. J. (2012). Mycorrhizainduced resistance and priming of plant defenses. Journal of chemical ecology, 38(6):651664.

Kang, Y., Clark, R., Makiyama, M., and Fewell, J. (2011). Mathematical modeling on obligate mutualism: Interactions between leaf-cutter ants and their fungus garden. Journal of theoretical biology, 289:116-127.

Kerr, B., Riley, M. A., Feldman, M. W., and Bohannan, B. J. (2002). Local dispersal promotes biodiversity in a real-life game of rock-paper-scissors. Nature, 418(6894):171.

Kiers, E. T., Duhamel, M., Beesetty, Y., Mensah, J. A., Franken, O., Verbruggen, E., Fellbaum, C. R., Kowalchuk, G. A., Hart, M. M., Bago, A., et al. (2011). Reciprocal rewards stabilize cooperation in the mycorrhizal symbiosis. science, 333(6044):880-882.

Kiers, E. T. and Van Der Heijden, M. G. (2006). Mutualistic stability in the arbuscular mycorrhizal symbiosis: exploring hypotheses of evolutionary cooperation. Ecology, 87(7):16271636.

Klironomos, J. N. and Hart, M. M. (2002). Colonization of roots by arbuscular mycorrhizal fungi using different sources of inoculum. Mycorrhiza, 12(4):181-184.

Knowlton, N. and Rohwer, F. (2003). Multispecies microbial mutualisms on coral reefs: the host as a habitat. the american naturalist, 162(S4):S51-S62.

Koch, A. M., Antunes, P. M., Barto, E. K., Cipollini, D., Mummey, D. L., and Klironomos, J. N. (2011). The effects of arbuscular mycorrhizal (am) fungal and garlic mustard introductions on native am fungal diversity. Biological Invasions, 13(7):1627-1639.

Kot, M. (2001). Elements of mathematical ecology. Cambridge University Press.

Křivan, V. and Revilla, T. A. (2019). Plant coexistence mediated by adaptive foraging preferences of exploiters or mutualists. Journal of theoretical biology, 480:112-128. 
Latef, A. A. H. A., Hashem, A., Rasool, S., Abd_Allah, E. F., Alqarawi, A., Egamberdieva, D., Jan, S., Anjum, N. A., and Ahmad, P. (2016). Arbuscular mycorrhizal symbiosis and abiotic stress in plants: A review. Journal of plant biology, 59(5):407-426.

Lekberg, Y. and Koide, R. T. (2014). Integrating physiological, community, and evolutionary perspectives on the arbuscular mycorrhizal symbiosis. Botany, 92(4):241-251.

Levine, J. M., Bascompte, J., Adler, P. B., and Allesina, S. (2017). Beyond pairwise mechanisms of species coexistence in complex communities. Nature, 546(7656):56.

Maherali, H. and Klironomos, J. N. (2007). Influence of phylogeny on fungal community assembly and ecosystem functioning. science, 316(5832):1746-1748.

Martignoni, M. M., Hart, M. M., Tyson, R. C., and Garnier, J. (2020). Diversity within mutualist guilds promotes coexistence and reduces the risk of invasion from an alien mutualist. Proceedings of the Royal Society B, 287(1923):20192312.

May, R. M. (1972). Will a large complex system be stable? Nature, 238(5364):413.

May, R. M. (1976). Models for two interacting populations. Theoretical ecology: principles and applications, pages 49-70.

Mayfield, M. M. and Stouffer, D. B. (2017). Higher-order interactions capture unexplained complexity in diverse communities. Nature ecology $\&$ evolution, 1(3):0062.

McCann, K. S. (2000). The diversity-stability debate. Nature, 405(6783):228.

Miki, T. (2012). Microbe-mediated plant-soil feedback and its roles in a changing world. Ecological Research, 27(3):509-520.

Moeller, H. V. and Neubert, M. G. (2016). Multiple friends with benefits: an optimal mutualist management strategy? The American Naturalist, 187(1):E1-E12.

Molbo, D., Machado, C. A., Sevenster, J. G., Keller, L., and Herre, E. A. (2003). Cryptic species of fig-pollinating wasps: implications for the evolution of the fig-wasp mutualism, sex allocation, and precision of adaptation. Proceedings of the National Academy of Sciences, 100(10):5867-5872.

Morales, M. A. (2011). Model selection analysis of temporal variation in benefit for an anttended treehopper. Ecology, 92(3):709-719.

Morris, W. F., Vázquez, D. P., and Chacoff, N. P. (2010). Benefit and cost curves for typical pollination mutualisms. Ecology, 91(5):1276-1285.

Mummey, D. L., Antunes, P. M., and Rillig, M. C. (2009). Arbuscular mycorrhizal fungi pre-inoculant identity determines community composition in roots. Soil Biology and Biochemistry, 41(6):1173-1179.

Murray, J. D. (2007). Mathematical biology: I. An introduction, volume 17. Springer Science \& Business Media.

Öpik, M., Moora, M., Liira, J., and Zobel, M. (2006). Composition of root-colonizing arbuscular mycorrhizal fungal communities in different ecosystems around the globe. Journal of Ecology, 94(4):778-790. 
Palmer, T. M., Stanton, M. L., and Young, T. P. (2003). Competition and coexistence: exploring mechanisms that restrict and maintain diversity within mutualist guilds. the american naturalist, 162(S4):S63-S79.

Parniske, M. (2008). Arbuscular mycorrhiza: the mother of plant root endosymbioses. Nature Reviews Microbiology, 6(10):763.

Pearson, J. and Jakobsen, I. (1993). Symbiotic exchange of carbon and phosphorus between cucumber and three arbuscular mycorrhizal fungi. New phytologist, 124(3):481-488.

Pellmyr, O. (1999). Systematic revision of the yucca moths in the tegeticula yuccasella complex (lepidoptera: Prodoxidae) north of mexico. Systematic Entomology, 24(3):243-271.

Peterson, R. L. and Guinel, F. C. (2000). The use of plant mutants to study regulation of colonization by am fungi. In Arbuscular mycorrhizas: physiology and function, pages 147-171. Springer.

Powell, J. R. and Bennett, A. E. (2016). Unpredictable assembly of arbuscular mycorrhizal fungal communities. Pedobiologia, 59(1-2):11-15.

Ravnskov, S. and Jakobsen, I. (1995). Functional compatibility in arbuscular mycorrhizas measured as hyphal p transport to the plant. New Phytologist, 129(4):611-618.

Rygiewicz, P. T. and Andersen, C. P. (1994). Mycorrhizae alter quality and quantity of carbon allocated below ground. Nature, 369(6475):58.

Sawers, R. J., Svane, S. F., Quan, C., Grønlund, M., Wozniak, B., Gebreselassie, M.-N., González-Muñoz, E., Montes, R. A. C., Baxter, I., Goudet, J., et al. (2017). Phosphorus acquisition efficiency in arbuscular mycorrhizal maize is correlated with the abundance of root-external hyphae and the accumulation of transcripts encoding pht1 phosphate transporters. New Phytologist, 214(2):632-643.

Schoener, T. W. (1974). Some methods for calculating competition coefficients from resourceutilization spectra. The American Naturalist, 108(961):332-340.

Smith, G. R., Steidinger, B. S., Bruns, T. D., and Peay, K. G. (2018). Competitioncolonization tradeoffs structure fungal diversity. The ISME journal, 12(7):1758.

Smith, S. E. and Read, D. J. (2010). Mycorrhizal symbiosis. Academic press.

Smith, S. E. and Smith, F. A. (2012). Fresh perspectives on the roles of arbuscular mycorrhizal fungi in plant nutrition and growth. Mycologia, 104(1):1-13.

Smith, S. E., Smith, F. A., and Jakobsen, I. (2003). Mycorrhizal fungi can dominate phosphate supply to plants irrespective of growth responses. Plant physiology, 133(1):16-20.

Stanton, M. L. (2003). Interacting guilds: moving beyond the pairwise perspective on mutualisms. The American Naturalist, 162(S4):S10-S23.

Sugiyama, A., Ueda, Y., Zushi, T., Takase, H., and Yazaki, K. (2014). Changes in the bacterial community of soybean rhizospheres during growth in the field. PloS one, 9(6).

Sylvia, D. M. and Williams, S. E. (1992). Vesicular-arbuscular mycorrhizae and environmental stress. Mycorrhizae in sustainable agriculture, (mycorrhizaeinsu):101-124. 
Thomson, B., Robson, A., and Abbott, L. (1990). Mycorrhizas formed by gigaspora calospora and glomus fasciculatum on subterranean clover in relation to soluble carbohydrate concentrations in roots. New Phytologist, 114(2):217-225.

Treseder, K. K. (2013). The extent of mycorrhizal colonization of roots and its influence on plant growth and phosphorus content. Plant and Soil, 371(1-2):1-13.

Treseder, K. K. and Cross, A. (2006). Global distributions of arbuscular mycorrhizal fungi. Ecosystems, 9(2):305-316.

Valdovinos, F. S., Moisset de Espanés, P., Flores, J. D., and Ramos-Jiliberto, R. (2013). Adaptive foraging allows the maintenance of biodiversity of pollination networks. Oikos, 122(6):907-917.

van Aarle, I. M. and Olsson, P. A. (2003). Fungal lipid accumulation and development of mycelial structures by two arbuscular mycorrhizal fungi. Applied and Environmental Microbiology, 69(11):6762-6767.

Van der Heijden, M. G., Klironomos, J. N., Ursic, M., Moutoglis, P., Streitwolf-Engel, R., Boller, T., Wiemken, A., and Sanders, I. R. (1998). Mycorrhizal fungal diversity determines plant biodiversity, ecosystem variability and productivity. Nature, 396(6706):69.

Van der Putten, W. H., Bardgett, R. D., Bever, J. D., Bezemer, T. M., Casper, B. B., Fukami, T., Kardol, P., Klironomos, J. N., Kulmatiski, A., Schweitzer, J. A., et al. (2013). Plant-soil feedbacks: the past, the present and future challenges. Journal of Ecology, 101(2):265-276.

Vandermeer, J. H. and Boucher, D. H. (1978). Varieties of mutualistic interaction in population models. Journal of Theoretical Biology, 74(4):549-558.

Vierheilig, H., Bago, B., Lerat, S., and Piché, Y. (2002). Shoot-produced, light-dependent factors are partially involved in the expression of the arbuscular mycorrhizal (am) status of am host and non-host plants. Journal of Plant Nutrition and Soil Science, 165(1):21-25.

Walder, F., Niemann, H., Mathimaran, N., Lehmann, M. F., Boller, T., and Wiemken, A. (2012). Mycorrhizal networks: common goods of plants shared under unequal terms of trade. Plant physiology, pages pp-112.

Walder, F. and van der Heijden, M. G. (2015). Regulation of resource exchange in the arbuscular mycorrhizal symbiosis. Nature Plants, 1(11):15159.

Wang, W., Shi, J., Xie, Q., Jiang, Y., Yu, N., and Wang, E. (2017). Nutrient exchange and regulation in arbuscular mycorrhizal symbiosis. Molecular plant, 10(9):1147-1158.

Wilson, W., Morris, W., and Bronstein, J. L. (2003). Coexistence of mutualists and exploiters on spatial landscapes. Ecological monographs, 73(3):397-413.

Wright, D. H. (1989). A simple, stable model of mutualism incorporating handling time. The American Naturalist, 134(4):664-667.

Zhu, Y.-G. and Miller, R. M. (2003). Carbon cycling by arbuscular mycorrhizal fungi in soil-plant systems. Trends in plant science, 8(9):407-409. 


\section{Supplementary information}

\section{Analysis of the one plant - one fungus system}

Conditions for the existence of coexistence steady states: We are interested in deriving conditions for the existence of steady states for which plant and AM fungi coexistence is observed. To facilitate computations, we reduce the number of model parameters in Eq. (1) and non-dimensionalise the equations by carrying out the substitutions given in Table S2. We consider a version of the model for which $r_{p}=0$ and maintenance costs are non-linearl (i.e., $n=2$ and $s=2$ ). Insights on the cases $r_{p}>0$ and $n, s \neq 2$ will be given below (see Figs. S4, S5 and S6). We obtain the non-dimensional model

$$
\begin{aligned}
\frac{d P}{d \tau} & =Q \frac{M}{D+P} P-M P-\eta_{P} P^{2}, \\
\frac{d M}{d \tau} & =P M-\frac{P}{D+P} M-\eta_{M} M^{2} .
\end{aligned}
$$

Nullclines of Eq. (5) can be found by setting $d P / d \tau$ and $d M / d \tau$ to zero and solving for $P$ and $M$. We obtain

$$
\begin{aligned}
\frac{d P}{d \tau}=0 & \Longleftrightarrow \quad P=0 \quad \text { or } \quad M=\frac{\eta_{P} P(D+P)}{Q-D-P}, \\
\frac{d M}{d \tau}=0 & \Longleftrightarrow M=0 \quad \text { or } \quad M=\frac{P(P+D-1)}{\eta_{M}(D+P)} .
\end{aligned}
$$

The first nullcline in Eq. (6a) and in Eq. (6b) correspond to the absence of AM fungi and plant biomass respectively, and to the horizontal and vertical axes in the phase plane. The origin is therefore always a steady state.

The second nullcline of Eq. (6a) is an hyperbola with vertical asymptote at $P=Q-D$ (Fig. S1). In order for Eq. (6a) to allow positive values for $M$ and $P$ the asymptote must be in the positive quadrant, i.e. we require $Q-D>0$. This requirement yields us the first condition for the existence of a steady state, which can be expressed in terms of the original parameters as

$$
\alpha>\frac{q_{c p}}{q_{h p}} \beta d .
$$

Expression (7) essentially states that for a coexistence steady state to occur, the ability of the fungi to supply phosphorus to the plant $(\alpha)$ must be sufficiently large. At the same time, the ratio between the conversion factors of nutrients given to received into plant biomass $\left(q_{h p} / q_{c p}\right)$ and the carbon sink strength $(\beta)$ must be small enough. Moreover $d$ must be small, i.e., the threshold of plant biomass that determines when the benefit of the mutualism for the plant is limited by the plant or by the fungal biomass must be relatively low.

The second nullcline of Eq. (6b) is also an hyperbola with asymptote at $-D$. Intersections of the hyperbola with the horizontal axis occur at $P=0$ and $P=1-D$. If $D>1$, the

Table S1: Variables and parameters of the model given in Eq. (1), with respective measurement units.

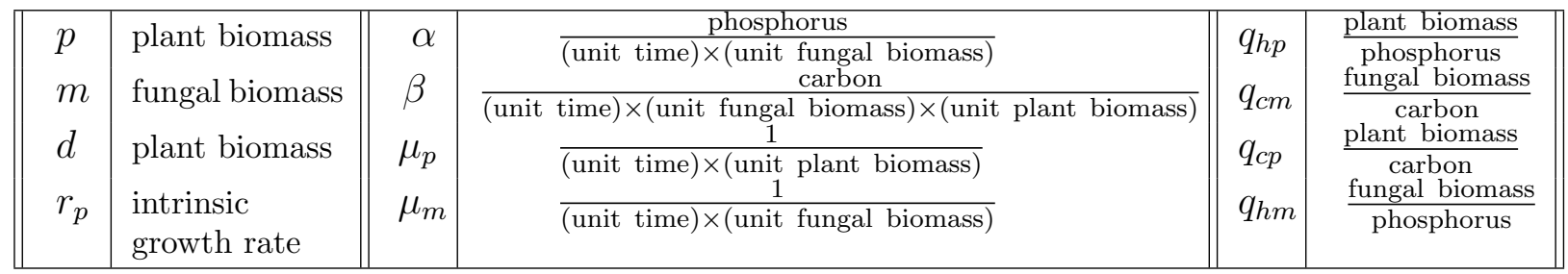


Table S2: Description of the non-dimensional parameters used to translate Eq. (1) into Eq. (5) .

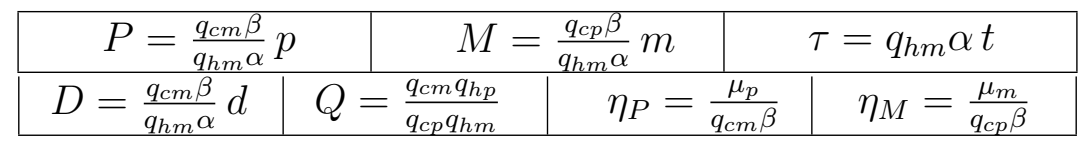

hyperbola assumes values in the positive quadrant for $P>0$, while if $D<1$ the hyperbola assumes values in the first quadrant for $P>1-D$ (Fig. S1). In order for the nullclines in Eqs. (6a) and (6b) to intersect, the asymptote at $P=Q-D$ in (6a) must occur at $P>1-D$. This condition gives a second requirement for the existence of a steady state, that is $Q-D>1-D \Leftrightarrow Q>1$. In term of the original parameters, this condition translates to

$$
\frac{q_{c m} q_{h p}}{q_{c p} q_{h m}}>1
$$

Eq. (8) states that the product of the conversion factors of the nutrients received, i.e., carbon for the fungi and phosphorus for the plant, must be larger than the conversion factors of the nutrients given.

An expression for the values of $P^{*}$ at steady state can be derived by setting Eqs. (6a) and (6b) equal to each other and finding the roots of the polynomial

$$
\eta_{P} \eta_{M}\left(P^{*}+D\right)^{2}-\left(Q-D-P^{*}\right)\left(P^{*}+D-1\right)=0 .
$$

Steady states occur at

$$
P_{1,2}^{*}=\frac{Q+1 \pm \sqrt{(Q-1)^{2}-4 Q \eta_{P} \eta_{M}}}{2\left(1+\eta_{P} \eta_{M}\right)}-D
$$

The steady states $P^{*}$ assume real values only if the expression under the square root is greater than zero. This requirement translates into a third condition for coexistence, i.e., $\eta_{P} \eta_{M}<(Q-1)^{2} / 4 Q$. In term of the original parameters this condition can be written as

$$
\mu_{p} \mu_{m}<\beta^{2} \frac{q_{c m} q_{h p}-q_{h m} q_{c p}}{4 q_{h m} q_{h p}} .
$$

Expression (11) essentially states that to allow stable coexistence of plant and AM fungi the maintenance rates for plant and $\operatorname{AMF}\left(\mu_{p}\right.$ and $\left.\mu_{m}\right)$ can not be too large.

Eq. (10) can have zero, one or two positive solutions depending on the choice of the parameters. The stability analysis conducted below and the graphical stability analysis of Fig. S1 show that when only one coexistence steady state exists (i.e., $P_{1}^{*}<0$ and $P_{2}^{*}>0$ ), it is always an attractor and the origin is a saddle. When two coexistence steady states exist the origin and $P_{2}^{*}$ are attractors and $P_{1}^{*}$ is a saddle, where $P_{1}^{*}<P_{2}^{*}$. In this case there are two distinct basins of attraction, one for the origin and one for the $P_{2}^{*}$ steady state. The biological interpretation of this case is that below certain thresholds of plant and fungal biomass, mutual growth can not be observed.

The steady state value of the plant (the positive root in Eq. (10)) corresponds to the nondimensionalised version of the upper bound to plant growth, and determines the maximal plant biomass at which plant growth saturates. This value increases with $Q$, and decreases with $D, \eta_{P}$ or $\eta_{M}$. Eq. (10) can be rewritten in terms of the original parameters as

$$
p_{1,2}^{*}=\frac{\frac{q_{h p} \alpha}{q_{c p}}+\frac{\alpha q_{h m}}{q_{c m}} \pm \frac{\alpha q_{h m}}{q_{c m}} \sqrt{\left(\frac{q_{h p} q_{c m}}{q_{c p} q_{h m}}-1\right)^{2}-4 \frac{q_{h p} \mu_{p} \mu_{m}}{q_{h m} q_{c p}^{2} \beta^{2}}}}{2\left(\beta+\frac{\mu_{p} \mu_{m}}{q_{c m} q_{c p} \beta}\right)}-d .
$$


We are interested in determining how variations in all of the model parameters affect the value of the stable steady states $p_{2}^{*}$ and $m_{2}^{*}$, representing the final biomass of the plant and of the AM fungi respectively (the stability analysis will be given in the following section). Plots in Fig. S2 shows variations of $p_{2}^{*}$ as a function of each parameter. From Eq. (6) one can derive an expression for the non-dimensional steady state $M_{2}^{*}$, that is

$$
M_{2}^{*}=\frac{\eta_{P} P_{2}^{*}\left(D+P_{2}^{*}\right)}{Q-D-P_{2}^{*}} .
$$

Or, in terms of the original parameters

$$
m_{2}^{*}=\frac{\mu_{p}}{\alpha q_{h m}} \frac{p_{2}^{*}\left(d+p_{2}^{*}\right)}{\left(\frac{q_{c p} \alpha}{q_{h p} \beta}-d-p_{2}^{*}\right)} .
$$

The plots in Fig. S3 show how $m_{2}^{*}$ varies as a function of the model parameters.
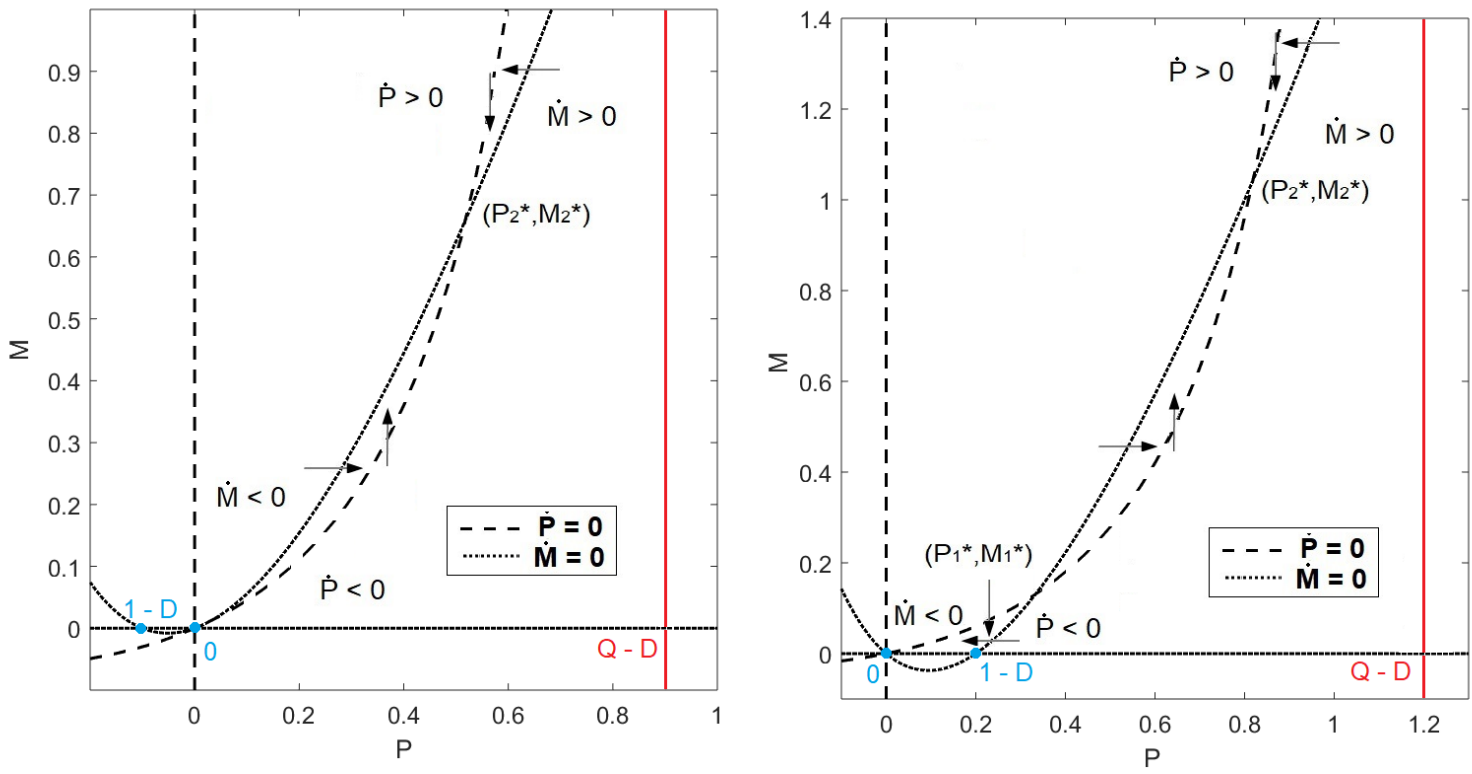

Fig. S1: Nullclines $\dot{P}=0$ (dashed line) and $\dot{M}=0$ (dotted line) of Eq. (1), where $Q=2, \eta_{P}=\eta_{M}=$ $0.3, N=1$ and $D=1.1$ (left panel) or $D=0.8$ (right panel). Blue dots represent the intersections of the nullcline $\dot{M}=0$ with the horizontal axis. When $D<1$ the non-zero interception occurs for $P>0$. When $D<1$ the non-zero interception occurs for $P<0$. Arrows represent qualitatively the direction of the flow on the nullclines and nearby the steady states. The steady states at $\left(P^{*}, M^{*}\right)$ (left panel) and $\left(P_{2}^{*}, M_{2}^{*}\right)$ (right panel) are always stable. The left panel represents the situation for which only one positive steady state exists, and the steady state at the origin is a saddle. The right panel corresponds to the case where two steady states occur. The steady state at $\left(P_{1}^{*}, M_{1}^{*}\right)$ is a saddle and the origin an attractor. The asymptote of the nullcline $\dot{P}=0$ occurring at $P=Q-D$ is shown in red. 

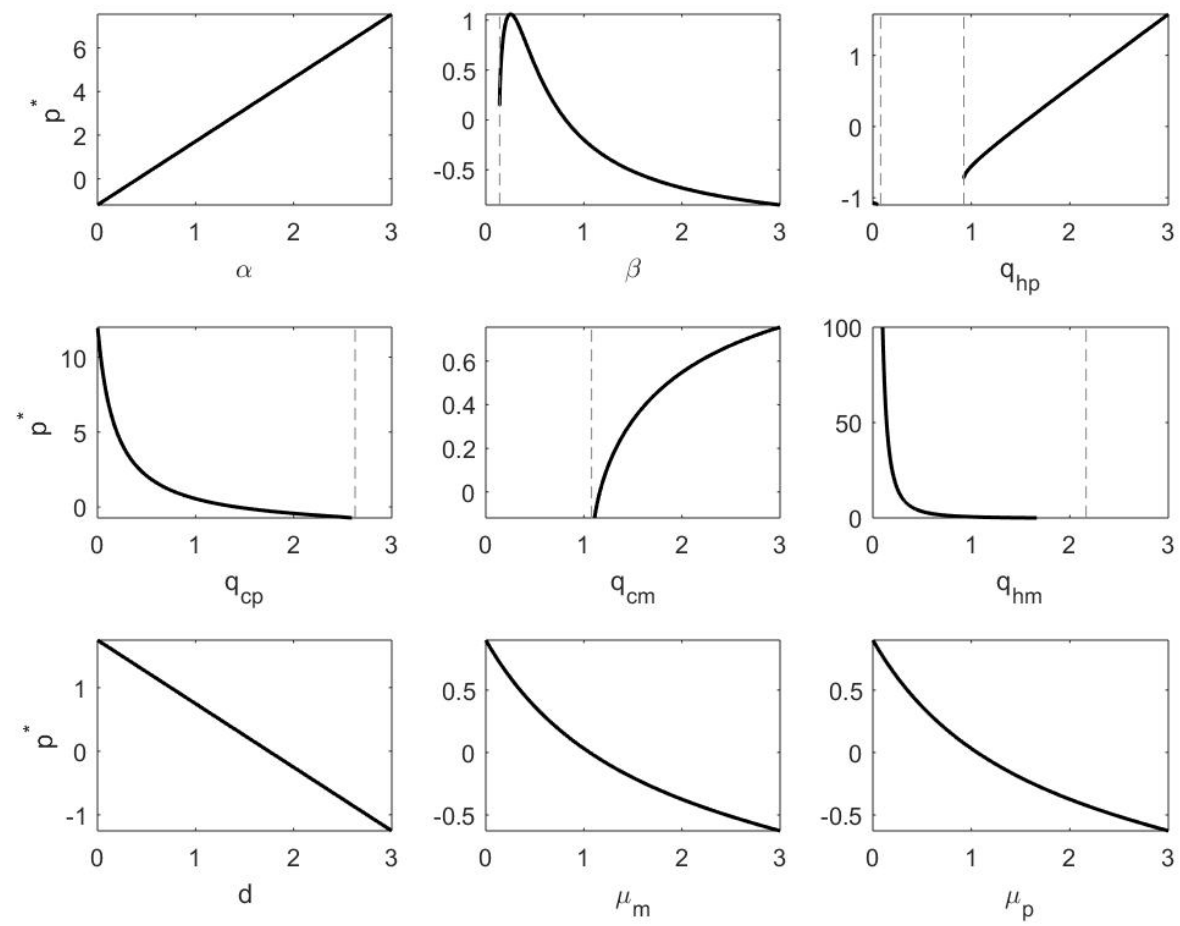

Fig. S2: Steady state value $p_{2}^{*}$ (or plant final biomass) as a function of the model parameters. Default parameter values are $q_{h p}=q_{c m}=2, q_{h m}=q_{c p}=1, \alpha=0.6, \beta=0.5, \mu_{m}=\mu_{p}=0.3, d=1.2$. 

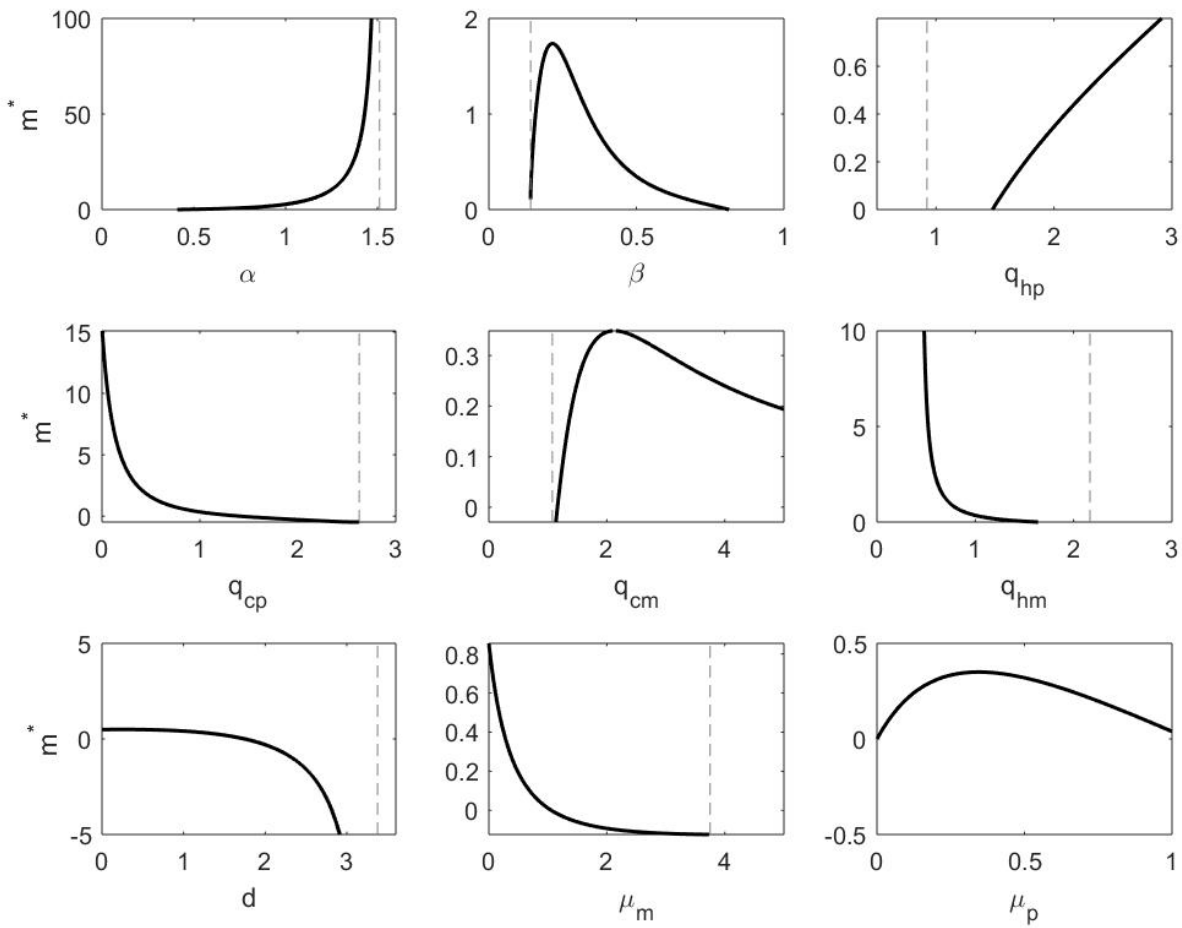

Fig. S3: Steady state value $m_{2}^{*}$ (or fungal final biomass) as a function of the model parameters. Default parameter values correspond to those in Fig. S2.

Stability of the coexistence steady states: I consider Eq. (5), describing variation in plant and fungal biomass over time, when the plant is associating with a single species of AM fungi. As shown in the previous section, as long as conditions (7), (8) and (11) are satisfied this system presents a minimum of one and a maximum of two coexistence steady states $\left(P_{1,2}^{*}, M_{1,2}^{*}\right)$. We are interested in determining the stability of these coexistence steady states. For practicality, we will initially refer to both steady states as $\left(P^{*}, M^{*}\right)$ and distinguish the two different cases toward the end of the analysis. At steady state, it holds that

$$
\begin{aligned}
& P^{*}\left(\frac{Q}{P^{*}+D}-1\right)=\frac{\eta_{P}\left(P^{*}\right)^{2}}{M^{*}}, \\
& M^{*}\left(1-\frac{1}{P^{*}+D}\right)=\frac{\eta_{M}\left(M^{*}\right)^{2}}{P^{*}} .
\end{aligned}
$$

Hence the Jacobian of Eq. (5) at steady state can be written as

$$
\begin{aligned}
J_{\left(P^{*}, M^{*}\right)} & =\left(\begin{array}{cc}
-\left(\frac{Q M^{*}}{\left(P^{*}+D\right)^{2}}+\eta_{P}\right) P^{*} & P^{*}\left(\frac{Q}{P^{*}+D}-1\right) \\
M^{*}\left(1-\frac{1}{P^{*}+D}\right)+\frac{P^{*} M^{*}}{\left(P^{*}+D\right)^{2}} & -\eta_{M} M^{*}
\end{array}\right) \\
& =\left(\begin{array}{cc}
-\left(\frac{Q M^{*}}{\left(P^{*}+D\right)^{2}}+\eta_{P}\right) P^{*} & \frac{\eta_{P}\left(P^{*}\right)^{2}}{M^{*}} \\
\frac{\eta_{m}\left(M^{*}\right)^{2}}{P^{*}}+\frac{P^{*} M^{*}}{\left(P^{*}+D\right)^{2}} & -\eta_{M} M^{*}
\end{array}\right)
\end{aligned}
$$


One can first observe that the trace of $J$ is always negative. The determinant of $J$ can be computed by using the relationships found in Eq. (15)

$$
\begin{aligned}
\operatorname{det}(J) & =\left(\frac{Q M^{*}}{\left(P^{*}+D\right)^{2}}+\eta_{P}\right) P^{*} \eta_{M} M^{*}-\frac{\eta_{P}\left(P^{*}\right)^{2}}{M^{*}}\left(\frac{\eta_{M}\left(M^{*}\right)^{2}}{P^{*}}+\frac{P^{*} M^{*}}{\left(P^{*}+D\right)^{2}}\right) \\
& =\frac{P^{*}}{\left(P^{*}+D\right)^{2}}\left(\eta_{m}\left(M^{*}\right)^{2} Q-\eta_{P}\left(P^{*}\right)^{2}\right) \\
& =\frac{P^{*}}{\left(P^{*}+D\right)^{2}}\left(P^{*} M^{*}\left(1-\frac{1}{P^{*}+D}\right) Q-P^{*} M^{*}\left(\frac{Q}{P^{*}+D}-1\right)\right) \\
& =\frac{\left(P^{*}\right)^{2} M^{*}}{\left(P^{*}+D\right)^{3}}\left((1+Q)\left(P^{*}+D\right)-2 Q\right) .
\end{aligned}
$$

The sign of $\operatorname{det}(J)$ depends therefore on the sign of $(1+Q)\left(P^{*}+D\right)-2 Q$, where the coexistence steady state $\left(P^{*}, M^{*}\right)$ is stable if $P^{*}+D \geq 2 Q /(1+Q)$, and it is a saddle point otherwise.

From the analysis conducted above, we know that $X=\left(P^{*}+D\right)$ are roots of the polynomial $T(X)=\eta_{P} \eta_{M} X^{2}-(Q-X)(X-1)$ (see Eq. (9)). I use $X^{+}$and $X^{-}$to denote the positive and negative root of $T(X)$, where

$$
X^{ \pm}=\frac{1+Q \pm \sqrt{(1-Q)^{2}-4 Q \eta_{P} \eta_{M}}}{2\left(1+\eta_{M} \eta_{P}\right)} .
$$

To determine the stability of the steady states it is sufficient to show that

$$
X^{-} \leq 2 Q /(1+Q) \leq X^{+} .
$$

To prove Eq. (19), we want to understand the location of $2 Q /(1+Q)$ with respect to the roots of the polynomial $T(X)$. For this purpose, we need to determine the sign of $T(2 Q / 1+Q)$. We obtain

$$
\begin{aligned}
T(2 Q /(1+Q)) & =\eta_{P} \eta_{M} \frac{4 Q^{2}}{(1+Q)^{2}}-\left(Q-\frac{2 Q}{(1+Q)}\right)\left(\frac{2 Q}{(1+Q)}-1\right) \\
& =\eta_{P} \eta_{M} \frac{4 Q^{2}}{(1+Q)^{2}}-\frac{Q(Q-1)}{(1+Q)} \frac{(Q-1)}{(1+Q)} \\
& =\left(4 \eta_{P} \eta_{M} Q-(Q-1)^{2}\right) \frac{Q}{(1+Q)^{2}} \\
& =-\Delta \frac{Q}{(1+Q)^{2}} \leq 0 .
\end{aligned}
$$

Because $T(X)$ is an upward parabola, Eq. (20) implies that Eq. (19) holds true. One can conclude that the coexistence steady state $\left(P_{2}^{*}, M_{2}^{*}\right)$ associated to $X^{+}$is stable $($as $\operatorname{Tr}(J)<0$ and $\operatorname{det}(J)>0$ ), and the steady state $\left(P_{1}^{*}, M_{1}^{*}\right)$ associated to $X^{-}$is a saddle point (as $\operatorname{Tr}(J)<0$ and $\operatorname{det}(J)<0)$.

\section{Facultative mutualism for the plant $\left(r_{p}>0\right)$ and variation in the maintenance costs $(n, s \neq 2)$}

The analysis conducted above considered the case where the intrinsic growth rate of the plant is zero $\left(r_{p}=0\right)$, and maintenance costs increase non-linearly with plant and fungal biomass $(s, n=2)$. Here we investigate the behaviour of systems where $r_{p}>0$ and $n, s \neq 2$. We consider the model

$$
\begin{aligned}
\frac{d p}{d t} & =q_{h p} \alpha m \frac{p}{d+p}-q_{c p} \beta m p+r_{p} p-\mu_{p} p^{n}, \\
\frac{d m}{d t} & =q_{c m} \beta p m-q_{h m_{i}} \alpha \frac{p}{d+p} m-\mu_{m} m^{s} .
\end{aligned}
$$


Nullclines of Eq. (21) for $s>1$ are given by:

$$
\begin{aligned}
& \dot{p}=0 \leftrightarrow m=\frac{\mu_{p} p^{n-1}-r_{p}}{\left(q_{h p} \frac{\alpha}{d+p}-q_{c p} \beta\right)}, \\
& \dot{m}=0 \leftrightarrow m=\sqrt[s-1]{\frac{1}{\mu_{m}}\left(q_{c m} \beta p-q_{h m} \alpha \frac{p}{d+p}\right)} .
\end{aligned}
$$

A graphical representation of the nullclines of Eq. (22) is given in Fig. S4, for the case where $r_{p}>0$, and in Figs. S5, for the cases $n=1$ and $s=2$ (left figure), $n=3$ and $s=2$ (middle figure) and $n=2$ and $s=3$ (right figure). We can see that for all these cases a stable steady state exists, where the phase planes qualitatively resemble Fig. S1 (considered in the analysis).

When $s=1$, the nullcline of Eq. (22b) corresponds to a vertical axis at

$$
p=-q_{c m} \beta d+q_{h m} \alpha+\mu_{m}+\sqrt{\left(q_{c m} \beta d-q_{h m} \alpha-\mu_{m}\right)^{2}+4 q_{c m} \beta \mu_{m} d}
$$

For $d$ large enough, the nullclines do not intersect (see Fig. S6, left panel), what causes fungal biomass growth unbounded, while plant growth is limited. For $d$ small enough, intersection of the nullclines results into an unstable steady state (see Fig. S6). This situation corresponds to the right panel of Fig. S1.

Therefore, graphically we can observe that plant-fungus coexistence is observed whether the plant maintenance cost increases linearly $(n>1)$ or non-linearly with plant biomass $(n=1)$, and for $r_{p}>0$. Without any kind of self-limitation $(s=1)$ fungal growth is unlimited, however also in this case plant-fungus coexistence is observed. Further analytical work should provide the exact parameter range for which nullclines intersection (and thus mutualism establishment) is observed when $r_{p}>0$ and $n, s \neq 2$. Conditions for mutualism establishment for the case $r_{p}=0$ and $n, s=2$ are derived in the analysis above (see Eq. (7), $(8)$, and (11)).

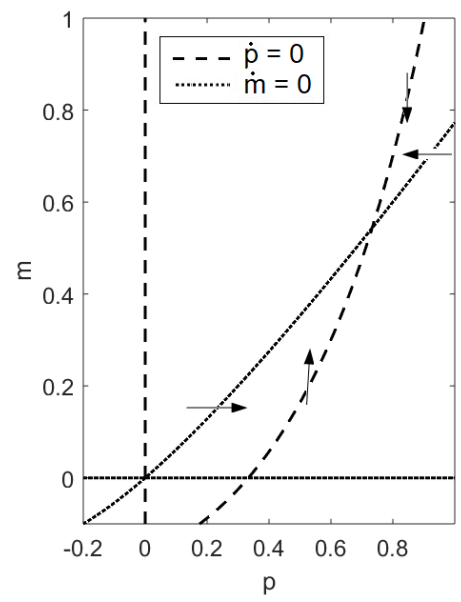

Fig. S4: Nullclines $\dot{p}=0$ (dashed line) and $\dot{p}=0$ (dotted line) of the system of differential equations given in Eq. (22) for $r_{p}>0$. Arrows represent qualitatively the direction of the flow nearby the stable steady state $\left(p^{*}, m^{*}\right)$. Parameter values are $r_{p}=0.1, q_{h p}=3, q_{c m}=2, q_{c p}=q_{h m}=1, \alpha=\beta=0.4$, $\mu_{m}=\mu_{p}=0.3, d=1.2, s=n=2$. 

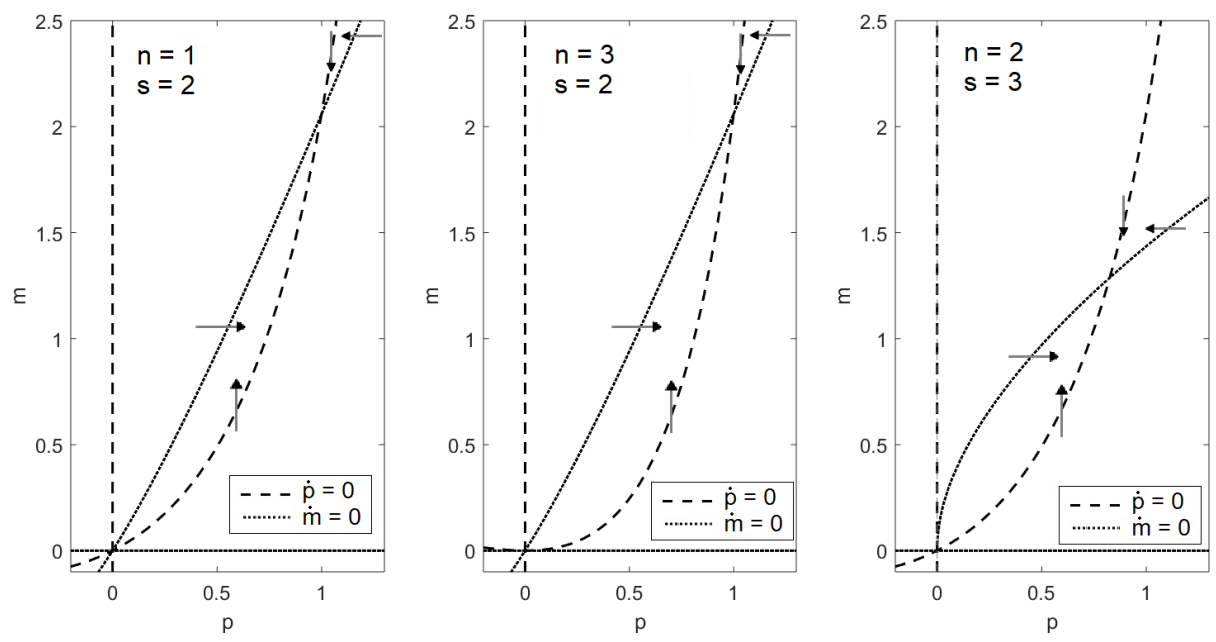

Fig. S5: Nullclines $\dot{p}=0$ (dashed line) and $\dot{p}=0$ (dotted line) of the system of differential equations given in Eq. (22) for different biomass dependence of the maintenance costs of the plant (parameter $n$ ) and the fungus (parameter $s$ ). Arrows represent qualitatively the direction of the flow nearby the stable steady state $\left(p^{*}, m^{*}\right)$. Parameter values are $n=1$ and $s=2$ (left figure), $n=3$ and $s=2$ (middle figure), and $n=2$ and $s=3$ (right figure). Other parameters corresponds to those of Fig. S4, except for $r_{p}=0$.
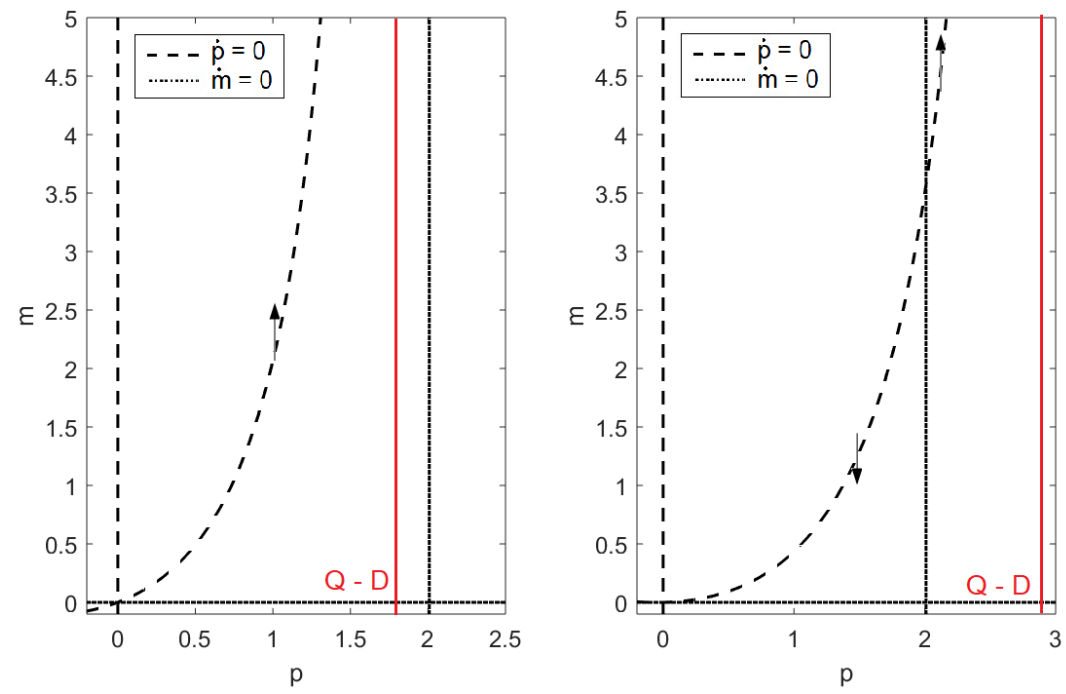

Fig. S6: Nullclines $\dot{p}=0$ (dashed line) and $\dot{p}=0$ (dotted line) of the system of differential equations given in Eq. (22) for linear maintenance cost of the fungus (parameter $s=1$ ). When $d$ is large enough, no coexistence steady state is observed (left figure) and fungal biomass increases unbounded. When $d$ is small enough, nullclines intersection results into an unstable steady state. Arrows represent qualitatively the direction of the flow nearby the stable steady state $\left(p^{*}, m^{*}\right)$. Parameter values are $n=2$ and $r_{p}=0$. Other parameters corresponds to those of Fig. S6.

\section{Analysis of the one plant - multiple fungi system}

Plant and fungal biomass as a function of the number of fungal species $N$ : Let us consider a system consisting of a plant $(p)$ and $N$ fungi $\left(m_{i}\right)$. I assume that all AM fungi have identical parameters. Then the non-dimensionalised model of Eq. (5) can be rewritten 
as

$$
\begin{aligned}
\frac{d P}{d \tau} & =\left[\left(\frac{Q}{D+P}-1\right) \sum_{i=1}^{N} M_{i}-\eta_{P} P\right] P, \\
\frac{d M_{i}}{d \tau} & =\left[\left(1-\frac{1}{D+P}\right) P-\eta_{M_{i}} M_{i}\right] M_{i} .
\end{aligned}
$$

The coexistence steady state are solutions to

$$
\begin{aligned}
M_{i}^{*} & =\frac{1}{\eta_{M_{i}}} P^{*} \frac{\left(D+P^{*}-1\right)}{D+P^{*}}, \\
\sum_{i=1}^{N} M_{i}^{*} & =\eta_{P} P^{*} \frac{\left(D+P^{*}\right)}{Q-D+P^{*}} .
\end{aligned}
$$

Since all $M_{i}$ are identical, Eq. (25) becomes

$$
\begin{aligned}
M_{i}^{*} & =\frac{1}{\eta_{M_{i}}} P^{*} \frac{\left(D+P^{*}-1\right)}{D+P^{*}}, \\
M_{i}^{*} & =\frac{\eta_{P}}{N} P^{*} \frac{\left(D+P^{*}\right)}{Q-D+P^{*}} .
\end{aligned}
$$

Combining those two equation one obtains an expression for the plant steady state

$$
P^{*}=\frac{Q+1 \pm \sqrt{(Q-1)^{2}-\frac{4 Q \eta_{P} \eta_{M_{i}}}{N}}}{2\left(1+\frac{\eta_{P} \eta_{M_{i}}}{N}\right)}-D .
$$

One can observe from Eq. (27) that $P^{*}$ is an increasing function of $N$, where the value of $P^{*}$ is bounded and approaches the asymptote $Q-D$ (see Fig. S1) as $N$ tends to infinity, i.e.,

$$
P^{*}(N \rightarrow \infty) \rightarrow Q-D .
$$

The value of the steady state $M_{i}^{*}$ is also increasing in $N$, where

$$
M_{i}^{*}=\frac{1}{\eta_{M_{i}}} P^{*}(N)\left(1-\frac{1}{D+P^{*}(N)}\right)
$$

and

$$
M_{i}^{*}(N \rightarrow \infty) \rightarrow \frac{Q-D}{\eta_{M_{i}}} \frac{Q-1}{Q} .
$$

Hence, the total fungal biomass tends to infinity for large $N$, i.e.,

$$
\sum_{i=1}^{N} M_{i}^{*}(N) \sim N \frac{Q-D}{\eta_{M_{i}}} \frac{Q-1}{Q} \rightarrow \infty \text { as } N \rightarrow \infty .
$$

The results of Eq. (31) can be explained by the fact that the model presented here does not take competition between fungi into account. Indeed competition should set a limit to fungal biomass at large fungal density. Extended versions of this model should consider this issue. 
Table S3: Description of the non-dimensional parameters used to translate Eq. (32a) into Eq. (37). These parameters correspond to the ones defined in Table 5, where the quantities $\alpha$ and $\beta$ have been substituted by $\bar{\alpha}$ and $\bar{\beta}$ (see Eq. (34)).

\begin{tabular}{|c|l|l|l|}
\hline \multicolumn{2}{|c|}{$P=\frac{q_{c m} \bar{\beta}}{q_{h m} \bar{\alpha}} p$} & $M_{i}=\frac{q_{c p} \beta_{i}}{q_{h m} \alpha_{i}} m_{i}$ & $\tau=q_{h m} \bar{\alpha} t$ \\
\hline$D=\frac{q_{c m} \bar{\beta}}{q_{h m} \bar{\alpha}} d$ & $Q=\frac{q_{c m} q_{h p}}{q_{c p} q_{h m}}$ & $\eta_{P}=\frac{\mu_{p}}{q_{c m} \bar{\beta}}$ & $\eta_{M_{i}}=\frac{\mu_{m_{i}}}{q_{c p} \beta_{i}}$ \\
\hline
\end{tabular}

Conditions for the existence of coexistence steady state: In this section, we will derive conditions for the existence of coexistence steady states in a system consisting of one plant $(p)$ and multiple AM fungi $\left(m_{i}\right.$, where $\left.i=1 . . N\right)$. Similarly to the previous section, for simplicity, we consider the case where the intrinsic growth rate of the plant is zero (i.e. $r_{p}=0$ ) and plant and fungi have non-linear maintenance costs $(n, s=2)$. We assume that different AM fungi have different resource exchange capacities $\alpha_{i}$ and $\beta_{i}$, and different maintenance rate $\mu_{m_{i}}$. The system of ordinary differential equations representing this situation is given by

$$
\begin{aligned}
\frac{d p}{d t} & =q_{h p} \sum_{i}\left(\alpha_{i} m_{i}\right) \frac{p}{d+p}-q_{c p} \sum_{i}\left(\beta_{i} m_{i}\right) p-\mu_{p} p^{2}, \\
\frac{d m_{i}}{d t} & =q_{c m_{i}} \beta_{i} p m_{i}-q_{h m_{i}} \alpha_{i} \frac{p}{d+p} m_{i}-\mu_{m_{i}} m_{i}^{2}, i=1 . . N .
\end{aligned}
$$

We are interested at looking at the steady state where there is coexistence of all species, $\left(p^{*}, m_{1}^{*}, \ldots, m_{N}^{*}\right)$, where the $m_{i}$ are solutions to

$$
m_{i}^{*}=\frac{p^{*}}{\mu_{m_{i}}}\left(q_{c m} \beta_{i}-\frac{q_{h m} \alpha_{i}}{d+p^{*}}\right) .
$$

I non-dimensionalise Eq. (33) by using the same substitutions given in Table 5 for $m_{i}$ and $\mu_{m_{i}}$. However, the non-dimensionalisation of $p$ and $d$ are expected to include the parameters of all fungi present. Hence we define

$$
\begin{aligned}
& \bar{\alpha}=\frac{1}{N} \sum_{i=1}^{N} \alpha_{i}, \\
& \bar{\beta}=\frac{1}{N} \sum_{i=1}^{N} \beta_{i} .
\end{aligned}
$$

The quantities $\bar{\alpha}$ and $\bar{\beta}$ correspond to the arithmetic mean of the resource exchange capacities $\alpha_{i}$ and $\beta_{i}$ of all AM fungi present. Eq. (34) can be used to non-dimensionalise Eq. (32a) by carrying on the substitutions illustrated in Table S3.

Eq. (33) can therefore be rewritten as

$$
M_{i}^{*}=\frac{1}{\eta_{M_{i}}} P^{*}\left(\frac{b_{i}}{a_{i}}-\frac{1}{P^{*}+D}\right),
$$

where $a_{i}=\alpha_{i} / \bar{\alpha}$ and $b_{i}=\beta_{i} / \bar{\beta}$.

From Eq. (35) one can see that in order for the steady state $M_{i}^{*}$ to be in the first quadrant, $b_{i} / a_{i}-1 /\left(P^{*}+D\right)$ needs to be positive. One can therefore derive a first condition for the existence of a coexistence steady state, that is

$$
P^{*}+D>\frac{a_{i}}{b_{i}}
$$


By substituting Eq. (35) and the parameters of Table S3 into Eq. (32a), we obtain that the value of the steady state $P^{*}$ is a solution to

$$
\sum_{i=1}^{N}\left(Q \frac{a_{i}}{b_{i}}-\left(P^{*}+D\right)\right)\left(\frac{b_{i}}{a_{i}}\left(P^{*}+D\right)-1\right) \frac{a_{i}}{\eta_{M_{i}}}-\eta_{P}\left(P^{*}+D\right)^{2}=0 .
$$

There must therefore exist $i_{0} \in 1, \ldots, N$ such that $Q a_{i_{0}} / b_{i_{0}}-D>0$, otherwise the polynomial of Eq. (37) is always negative for $P^{*}>0$. Hence a second condition for the existence of a coexistence steady state is that there must exist an $i_{0}$ such that

$$
\alpha_{i_{0}}>\frac{q_{c p}}{q_{h p}} d \beta_{i_{0}}
$$

This condition corresponds to Eq. (7) of the one plant - one fungus case. One can see that when multiple AM fungi are present, it is sufficient that one of the fungi satisfies Eq. (7). Combining the conditions given in Eqs. (36) and (38) one obtain

$$
Q \frac{a_{i_{0}}}{b_{i_{0}}}-\left(P^{*}+D\right)>Q \frac{a_{i_{0}}}{b_{i_{0}}}-\frac{a_{i_{0}}}{b_{i_{0}}}=\frac{a_{i_{0}}}{b_{i_{0}}}(Q-1)>0 .
$$

Hence we obtain the unique condition $Q>1$, or, in terms of the original parameters

$$
\frac{q_{c m} q_{h p}}{q_{h m} q_{c p}}>1
$$

which corresponds to condition (8), stated for the one plant - one fungus case.

By solving Eq. (37) we obtain an expression for the steady state $P^{*}$, that is

$$
P^{*}=\frac{(1+Q) \sum_{i=1}^{N} \frac{a_{i}}{\eta_{M_{i}}} \pm \sqrt{\Delta}}{2\left(\sum_{i=1}^{N} \frac{b_{i}}{\eta_{M_{i}}}+\eta_{P}\right)}-D
$$

with

$$
\Delta=(1+Q)^{2}\left(\sum_{i=1}^{N} \frac{a_{i}}{\eta_{M_{i}}}\right)^{2}-4 Q\left(\sum_{i=1}^{N} \frac{a_{i}^{2}}{b_{i} \eta_{M_{i}}}\right)\left(\sum_{i=1}^{N} \frac{b_{i}}{\eta_{M_{i}}}+\eta_{P}\right)
$$

We obtain therefore a third condition for the existence of a coexistence steady state, i.e.,

$$
\left(1+\frac{q_{c m} q_{h p}}{q_{c p} q_{h m}}\right)^{2}\left(\sum_{i=1}^{N} \frac{q_{c p} \alpha_{i} \beta_{i}}{\mu_{m_{i}}}\right)^{2}-4 \frac{q_{c m} q_{h p}}{q_{c p} q_{h m}}\left(\sum_{i=1}^{N} \frac{q_{c p} \alpha_{i}^{2}}{\mu_{m_{i}}}\right)\left(\sum_{i=1}^{N} \frac{q_{c p} \beta_{i}^{2}}{\mu_{m_{i}}}+\frac{\mu_{p}}{q_{c m}}\right)>0 .
$$

The expression of Eq. (43) involves the product and sum of the resource exchange capacities $\alpha_{i}$ and $\beta_{i}$. If we set $\mathbf{a}=\boldsymbol{\alpha} / \sqrt{\boldsymbol{\mu}_{m}}=\left(\alpha_{1} / \sqrt{\mu_{1}}, \ldots, \alpha_{n} / \sqrt{\mu_{n}}\right)$ and $\mathbf{b}=\boldsymbol{\beta} / \sqrt{\boldsymbol{\mu}}=$ $\left(\beta_{1} / \sqrt{\mu_{1}}, \ldots, \beta_{n} / \sqrt{\mu_{n}}\right)$, then we can rewrite condition (43) as follows:

$$
(1+Q)^{2}\left(1+\frac{\operatorname{Cov}(\mathbf{a}, \mathbf{b})}{\bar{a} \bar{b}}\right)^{2}-4 Q\left(1+\frac{\operatorname{Var}(\mathbf{a})}{\bar{a}^{2}}\right)\left(1+\frac{\mu_{p}}{N q_{c m} q_{c p} \bar{b}^{2}}+\frac{\operatorname{Var}(\mathbf{b})}{\bar{b}^{2}}\right)>0 .
$$

We can observe that condition (44) is more likely to be satisfied when the covariance between $\mathbf{a}$ and $\mathbf{b}$ and the means of $\mathbf{a}$ and $\mathbf{b}$ are large, and when the variances of $\mathbf{a}$ and $\mathbf{b}$ are small. Additionally, the condition is less likely to be satisfied when the maintenance cost of the fungi is large.

In sum, we can see that conditions for the existence of a coexistence steady state depend therefore on the resource exchange ability of the whole guild. Altogether, conditions (38), 
(40), and (44) suggest that as long as a good mutualist is present and the average mutualist quality of all species is large enough, a coexistence steady state exists.

Let us assume now that all fungi have the same resource exchange capacities $\alpha$ and $\beta$ and the same maintenance cost $\mu_{m}$, condition (44) reduces to

$$
\frac{\eta_{P} \eta_{M_{i}}}{N}<\frac{(1-Q)^{2}}{4 Q}
$$

what corresponds to condition (11) for the case where multiple AM fungi are present. This condition tells us that in the presence of multiple species, guild survival can be guaranteed even when maintenance costs are higher than what could be tolerated when only one fungal species is present.

Conditions for stability of the coexistence steady state: Let us consider a system consisting of one plant $(p)$ and two AM fungal species $\left(m_{1}\right.$ and $\left.m_{2}\right)$, differing only in their resource exchange capacities $\alpha_{i}$ and $\beta_{i}$. We write

$$
\begin{aligned}
\frac{d p}{d t} & =q_{h p}\left(\alpha_{1} m_{1}+\alpha_{2} m_{2}\right) \frac{p}{d+p}-q_{c p}\left(\beta_{1} m_{1}+\beta_{2} m_{2}\right) p-\mu_{p} p^{2}, \\
\frac{d m_{1}}{d t} & =q_{c m} \beta_{1} p m_{1}-q_{h m} \alpha_{1} \frac{p}{d+p} m_{1}-\mu_{m_{i}} m_{1}^{2}, \\
\frac{d m_{2}}{d t} & =q_{c m} \beta_{2} p m_{2}-q_{h m} \alpha_{2} \frac{p}{d+p} m_{2}-\mu_{m_{i}} m_{2}^{2} .
\end{aligned}
$$

We are interested at looking at the steady state $\left(p^{*}, m_{1}^{*}, m_{2}^{*}\right)$. The Jacobian of Eq. (46) at steady state is given by

$$
J_{\left(p^{*}, m_{1}^{*}, m_{2}^{*}\right)}=\left(\begin{array}{ccc}
\underbrace{-q_{h p}\left(\alpha_{1} m_{1}^{*}+\alpha_{2} m_{2}^{*}\right) \frac{p^{*}}{\left(d+p^{*}\right)^{2}}-\mu_{p} p^{*}}_{:=-A} & \underbrace{q_{h p} \alpha_{1} \frac{p^{*}}{d+p^{*}}-q_{c p} \beta_{1} p^{*}}_{:=C_{1}} & \underbrace{q_{h p} \alpha_{2} \frac{p^{*}}{d+p^{*}}-q_{c p} \beta_{2} p^{*}}_{:=C_{2}} \\
\underbrace{m_{1}^{*}\left(q_{c m} \beta_{1}-\frac{q_{h m} \alpha_{1}}{d+p^{*}}+q_{h m} \alpha_{1} \frac{p^{*}}{\left(d+p^{*}\right)^{2}}\right)}_{:=B_{1}} & -\mu_{m} m_{1}^{*} & 0 \\
\underbrace{m_{2}^{*}\left(q_{c m} \beta_{2}-\frac{q_{h m} \alpha_{2}}{d+p^{*}}+q_{h m} \alpha_{2} \frac{p^{*}}{\left(d+p^{*}\right)^{2}}\right)}_{:=B_{2}} & 0 & -\mu_{m} m_{2}^{*}
\end{array}\right) .
$$

We know that $A>0, B_{1}>0$, and $B_{2}>0$ (see Eq. (33)). The sign of $C_{1}$ and $C_{2}$ is unknown, however we can notice that these terms increase for increasing $\alpha_{i}$ and decrease for increasing $\beta_{i}$. We can therefore say that $C_{i}$ (where $i=1,2$ ) increase for increasing resource exchange capacity of the fungal species. The characteristic polynomial of Eq. (47) is given by

$$
\begin{aligned}
P(\lambda)= & \lambda^{3}+\lambda^{2} \underbrace{\mu_{m}\left(m_{1}^{*}+m_{2}^{*}\right)+A}_{:=a_{1}}+\lambda \underbrace{\left(\mu_{m}^{2} m_{1}^{*} m_{2}^{*}+\mu_{m}\left(m_{1}^{*}+m_{2}^{*}\right)-B_{1} C_{1}-B_{2} C_{2}\right)}_{:=a_{3}} \\
& +\underbrace{A \mu_{m}^{2} m_{1}^{*} m_{2}^{*}-\mu_{m} m_{1}^{*} B_{2} C_{2}-\mu_{m} m_{2}^{*} B_{1} C_{1}=0}_{:=a_{2}} .
\end{aligned}
$$

According to the Routh-Hurwitz conditions for stability (Murray, 2007), in order for $\operatorname{Re}(\lambda)>0$ the following three conditions need to be satisfied

$$
\text { (i) } a_{1}>0, \quad \text { (ii) } a_{3}>0, \quad \text { and }(\text { iii }) a_{1} a_{2}-a_{3}>0 \text {. }
$$


One can directly see that $a_{1}>0$, i.e., $(i)$ is always satisfied. Hence we obtain the following two conditions for stability of the coexistence steady state

(ii) $A \mu_{m} m_{1}^{*} m_{2}^{*}-m_{1}^{*} B_{2} C_{2}-m_{2}^{*} B_{1} C_{1}>0$,

(iii) $\mu_{m}\left(m_{1}^{*}+m_{2}^{*}\right)\left(\mu_{m}^{2} m_{1}^{*} m_{2}^{*}+\mu_{m}\left(m_{1}^{*}+m_{2}^{*}\right)+A\right)-B_{1} C_{1}\left(A+\mu_{m} m_{1}^{*}\right)-B_{2} C_{2}\left(A+\mu_{m} m_{2}^{*}\right)>0$.

We want now to show that condition $(i i)$ is always satisfied as well. We first reformulate the expression for $a_{3}$ as follows:

$a_{3}=\mu_{m}^{2} m_{1}^{*} m_{2}^{*}\left(\mu_{p} p^{*}-\sum_{i} \frac{m_{i}^{*} C_{i}}{p^{*}}\right)+\frac{\mu_{m}^{2} m_{1}^{*} m_{2}^{*} p^{*}}{p^{*}+d}\left(q_{h p}\left(\alpha_{1} m_{1}^{*}+\alpha_{2} m_{2}^{*}\right)-q_{h m}\left(\frac{\alpha_{1} C_{1}}{\mu_{m}}+\frac{\alpha_{2} C_{2}}{\mu_{m}}\right)\right)$.

The first term is equal to 0 , as from the one plant - one fungus model we know that

$$
P^{*}\left(M^{*}\left(\frac{Q}{P^{*}+D}-1\right)-\eta_{p} P^{*}\right)=0 \leftrightarrow P^{*}\left(\frac{Q}{P^{*}+D}-1\right)=\frac{\eta_{p}\left(P^{*}\right)^{2}}{M^{*}} .
$$

To do so we look at the eigenvalues of the linearized problem around this steady Additionally from

$$
M^{*}\left(P^{*}\left(1-\frac{1}{P^{*}+D}\right)-\eta_{m} M^{*}\right)=0 \leftrightarrow M^{*}\left(1-\frac{1}{P^{*}+D}\right)=\frac{\eta_{m}\left(M^{*}\right)^{2}}{P^{*}}
$$

and using the definition of $C_{i}$ we get

$$
\begin{aligned}
a_{3} & =\frac{\mu_{m}^{2} m_{1}^{*} m_{2}^{*} p^{*}}{p^{*}+d}\left(q_{h p}\left(q_{c m}\left(\alpha_{1} \beta_{1}+\alpha_{2} \beta_{2}\right)-q_{h m} \frac{\alpha_{1}^{2}+\alpha_{2}^{2}}{\left(p^{*}+d\right)}\right)\right. \\
& \left.-q_{h m}\left(q_{h p} \frac{\alpha_{1}^{2}+\alpha_{2}^{2}}{\left(p^{*}+d\right)}-q_{c p}\left(\alpha_{1} \beta_{1}+\alpha_{2} \beta_{2}\right)\right)\right) \\
& =\frac{\mu_{m}^{2} m_{1}^{*} m_{2}^{*} p^{*}}{p^{*}+d}\left(\left(q_{h p} q_{c m}+q_{c p} q_{h m}\right)\left(\alpha_{1} \beta_{1}+\alpha_{2} \beta_{2}\right)-2 q_{h m} q_{h p} \frac{\alpha_{1}^{2}+\alpha_{2}^{2}}{\left(p^{*}+d\right)}\right) .
\end{aligned}
$$

Thus we can see that $a_{3}>0$ if and only if $p^{*}+d \geq \frac{2 \frac{q_{h p}}{q_{c p}} \sum_{i} \alpha_{i}^{2}}{(1+Q) \sum_{i} \alpha_{i} \beta_{i}}$ where $Q=\frac{q_{c m} q_{h p}}{q_{h m} q_{c p}}$. Then in the adimentional variable it means that

$$
P^{*}+D \geq X_{0}=\frac{2 Q \sum_{i} \frac{\alpha_{i}^{2}}{\bar{\alpha}^{2}}}{(1+Q) \sum_{i} \frac{\alpha_{i}}{\bar{\alpha}} \frac{\beta_{i}}{\bar{\beta}}}=\frac{2 Q \sum_{i} a_{i}^{2}}{(1+Q) \sum_{i} a_{i} b_{i}} .
$$

To prove that we only need to evaluate the polynomial $T$ at $X_{0}$. Since $P^{*}+D$ is a root of polynomial $T(X)$ defined in Eq. (37), we only need to compute the value $T\left(X_{0}\right)$. We can show that

$$
T\left(X_{0}\right)=\frac{\Delta Q \sum_{i} a_{i}^{2}}{(1+Q)^{2}\left(\sum_{i} a_{i} b_{i}\right)^{2}}>0 .
$$

Thus we can conclude that $P^{*}+D>X_{0}$ and the coefficient $a_{3}$ is positive.

Hence, conditions $(i)$ and $(i i)$ are always satisfied. As long as condition $(i i i)$, together with the conditions for existence of the coexistence steady state derived previously in (38), (40) and (44), a stable steady state of multi-species coexistence exists. Numerically, we observe that the conditions for stability are always satisfied for the range of parameters for which a coexistence steady state exists. 


\section{The effect of differences in the resource exchange capacities of the fungi}

Analytical insights: I want to investigate how differences in the phosphorus exchange ability of the mutualists impact plant growth and the growth of other guild members. I will consider a guild of mutualists having different phosphorus exchange abilities (parameter $\alpha_{i}$ ), while all other parameters are held constant across all fungi.

Impact on plant biomass: I want to investigate how the value of the plant at steady state $\left(p^{*}\right)$ depends on the phosphorus exchange ability of all species present (i.e., how $p^{*}$ depends on $\left.\boldsymbol{\alpha}=\left(\alpha_{1}, \alpha_{2}, \ldots, \alpha_{N}\right)\right)$. For this purpose, we consider a plant $(p)$ associating with multiple fungal species $\left(m_{i}\right)$, differing in their ability to transfer phosphorus $\left(\alpha_{i}\right)$, and I compare the biomass of the plant at steady state $\left(p^{*}\right)$ when the plant is associating with $\mathrm{N}$ identical fungi (i.e., $\alpha_{i}=\alpha$ ), to the biomass of the plant at steady state when the plant is associating with $\mathrm{N}$ fungi differing in their resource exchange capacities. When multiple species are present, we know from Eq. (41) that the plant steady state corresponds to

$$
\begin{aligned}
& p^{*}(\boldsymbol{\alpha})=\frac{q_{h m} \bar{\alpha}}{q_{c m} \beta} P^{*}(\boldsymbol{\alpha})-d \\
& \text { with } P^{*}(\boldsymbol{\alpha})=\frac{1+Q+\sqrt{(1+Q)^{2}-4 Q \frac{1}{N} \sum_{i=1}^{N} a_{i}^{2}\left(1+\frac{\eta_{P} \eta_{M}}{N}\right)}}{2\left(1+\frac{\eta_{P} \eta_{M}}{N}\right)} .
\end{aligned}
$$

Considering that $\sum_{i} \alpha_{i}=N \bar{\alpha}$ and $\sum_{i} a_{i}=N$, the term $\sum_{i}^{N} a_{i}^{2}$ can be written as

$$
\frac{1}{N} \sum_{i=1}^{N} a_{i}^{2}=\frac{1}{N} \sum_{i=1}^{N}\left(a_{i}-\frac{1}{N} \sum_{i} a_{i}\right)^{2}+\left(\frac{1}{N} \sum_{i} a_{i}\right)^{2}=\frac{\operatorname{Var}(\boldsymbol{\alpha})}{\bar{\alpha}^{2}}+1,
$$

where $\operatorname{Var}(\boldsymbol{\alpha})$ is the variance of $\boldsymbol{\alpha}$, defined by

$$
\operatorname{Var}(\boldsymbol{\alpha})=\frac{1}{N} \sum_{i=1}^{N}\left(\alpha_{i}-\bar{\alpha}\right)^{2} \quad \text { with } \quad \bar{\alpha}=\frac{1}{N} \sum_{i=1}^{N} \alpha_{i}
$$

The value of the plant at steady state can be rewritten as

$$
\begin{aligned}
& p^{*}(\boldsymbol{\alpha})=\frac{q_{h m} \bar{\alpha}}{q_{c m} \beta} P^{*}(\boldsymbol{\alpha})-d \\
& \text { with } P^{*}(\boldsymbol{\alpha})=\frac{1+Q+\sqrt{(1+Q)^{2}-4 Q\left(1+\frac{\operatorname{Var}(\boldsymbol{\alpha})}{\bar{\alpha}^{2}}\right)\left(1+\frac{\eta_{P} \eta_{M}}{N}\right)}}{2\left(1+\frac{\eta_{P} \eta_{M}}{N}\right)} .
\end{aligned}
$$

Hence the final size of the plant $p^{*}(\boldsymbol{\alpha})$ depends on the mean as well as the variance of the phosphorus exchange capacities of all mutualists present. One can directly observe that $p^{*}(\boldsymbol{\alpha})$ decreases with respect to the variance. Additionally, if the AM fungi are identical (i.e., $\operatorname{Var}(\boldsymbol{\alpha})=0), p^{*}$ increases linearly with the value of $\bar{\alpha}$, and

$$
p^{*}(\boldsymbol{\alpha}) \leq p^{*}((\bar{\alpha}, \ldots, \bar{\alpha})) \leq p^{*}\left(\left(\max \left(\alpha_{i}\right), \ldots, \max \left(\alpha_{i}\right)\right)\right) \text { for any } \boldsymbol{\alpha} \in(0, \infty)^{N} .
$$

To give more insights into the analysis, we consider the case $N=2$, where a plant is associating with two AM fungi. We want to compare the biomass of the plant and the total fungal biomass 
at steady state when the plant is associating with two individuals that are good mutualists (species 1), with two individuals that are less beneficial mutualists (species 2), or with a combination of species 1 and species 2 . I assume that species 1 is a better mutualist with respect to species 2 , with $\alpha_{1}>\alpha_{2}$. We define $\alpha_{1}=\alpha+\epsilon$ and $\alpha_{2}=\alpha$, where $\epsilon \in(0, \infty)$. In this case the variance and mean of the resource exchange capacities can be computed as

$$
\bar{\alpha}=\alpha+\frac{\epsilon}{2} \text { and } \operatorname{Var}(\boldsymbol{\alpha})=\frac{\epsilon^{2}}{4},
$$

and the plant biomass at steady state is given by

$$
\begin{aligned}
p^{*}(\alpha, \alpha+\epsilon) & =\frac{q_{h m}(\alpha+\epsilon / 2)}{q_{c m} \beta} P^{*}(\epsilon)-d \\
\text { with } P^{*}(\epsilon) & =\frac{1+Q+\sqrt{(1+Q)^{2}-4 Q\left(1+\left(\frac{\epsilon / 2}{\alpha+\epsilon / 2}\right)^{2}\right)\left(1+\frac{\eta_{P} \eta_{M}}{2}\right)}}{2\left(1+\frac{\eta_{P} \eta_{M}}{2}\right)} .
\end{aligned}
$$

From Eq. (61) we already know that

$$
p^{*}(\alpha, \alpha+\epsilon)<p^{*}(\alpha+\epsilon / 2, \alpha+\epsilon / 2)<p^{*}(\alpha+\epsilon, \alpha+\epsilon) .
$$

Eq. (64) tells us that plant biomass at steady state is larger when the plant is associating with two individuals of species 1 , then when the plant is associating with a combination of species 1 and species 2 .

I want now to determine whether $p^{*}(\alpha, \alpha)$ is larger or smaller then $p^{*}(\alpha, \alpha+\epsilon)$, i.e., we want to understand if plant biomass at steady state is larger when the plant is associating with two individuals that are less beneficial mutualists (species 2), or with a combination of species 1 and species 2. I first consider the case where $\epsilon$ is large. For $\epsilon \rightarrow \infty$ we have,

$$
\begin{aligned}
& P^{*}(\epsilon) \sim \frac{1+Q+\sqrt{(1+Q)^{2}-4 Q\left(1+\eta_{P} \eta_{M}\right)-4 Q}}{2\left(1+\frac{\eta_{P} \eta_{M}}{2}\right)}=P_{\infty}^{*}>0, \\
& \text { and } p^{*}(\alpha, \alpha+\epsilon) \sim \frac{q_{h m}(\alpha+\epsilon / 2)}{q_{c m} \beta} P_{\infty}^{*}-d .
\end{aligned}
$$

Thus for $\epsilon$ large enough $P^{*}$ has a lower bound, however $p^{*}$ increases with increasing $\epsilon$. We can conclude that Eq. (67) holds true for $\epsilon$ large enough.

Let us consider now the case where $\epsilon$ is small. By using a Taylor expansion, we have the following estimates for plant biomass at steady state

$$
p^{*}(\alpha, \alpha+\epsilon)=p^{*}(\alpha, \alpha)+\frac{q_{h m}}{2 q_{c m} \beta} P^{*}(0) \epsilon+\mathcal{O}(\epsilon)
$$

One can conclude that also for small $\epsilon$ it holds

$$
p^{*}(\alpha, \alpha)<p^{*}(\alpha, \alpha+\epsilon) .
$$

Therefore

$$
p^{*}(\alpha, \alpha)<p^{*}(\alpha, \alpha+\epsilon)<p^{*}(\alpha+\epsilon, \alpha+\epsilon) \text { for any } \epsilon>0 .
$$

Eq. (68) states that the biomass of the plant at steady state is at highest when the plant is associating with a good mutualist (species 1), is at lowest when the plant is associating with a less beneficial mutualist (species 2) and it is between these two values when the plant is associating with a combination of good and less beneficial mutualists (species 1 and 2). 
Impact on fungal biomass: I consider now how differences in the phosphorus exchange ability of the fungi in a guild affect fungal biomass. The fungal biomass of each species at steady state is a function of the phosphorus exchange ability $\alpha$ and of plant biomass at steady state, and it is given by

$$
m_{\left(\alpha_{i}, p^{*}(\boldsymbol{\alpha})\right)}^{*}=\frac{p^{*}(\boldsymbol{\alpha})}{\mu_{m}}\left(q_{c m} \beta-\frac{q_{h m} \alpha_{i}}{d+p^{*}(\boldsymbol{\alpha})}\right),
$$

I want to understand if fungal biomass at steady state is increased or decreased in the presence of a species that has different phosphorus exchange ability. I assume that fungal species 1 has a higher phosphorus exchange ability that fungal species 2 , with $\alpha_{1}=\alpha+\epsilon$ and $\alpha_{2}=\alpha$. Let us first consider the fungal biomass of the more beneficial species (species 1) when the plant is associating with two good mutualists:

$$
\begin{aligned}
m_{\left(\alpha+\epsilon, p^{*}(\alpha+\epsilon, \alpha+\epsilon)\right)}^{*} & =\frac{p^{*}(\alpha+\epsilon, \alpha+\epsilon)}{\mu_{m}}\left(q_{c m} \beta-\frac{q_{h m}(\alpha+\epsilon)}{d+p^{*}(\alpha+\epsilon, \alpha+\epsilon)}\right) \\
& =\frac{p^{*}(\alpha+\epsilon, \alpha+\epsilon)}{\mu_{m}}\left(\frac{q_{c m} \beta\left(d+p^{*}(\alpha+\epsilon, \alpha+\epsilon)\right)-q_{h m}(\alpha+\epsilon)}{d+p^{*}(\alpha+\epsilon, \alpha+\epsilon)}\right) \\
& =\frac{p^{*}(\alpha+\epsilon, \alpha+\epsilon)}{\mu_{m}}\left(\frac{q_{h m}(\alpha+\epsilon)\left(P^{*}-1\right)}{d+p^{*}(\alpha+\epsilon, \alpha+\epsilon)}\right) .
\end{aligned}
$$

where $P^{*}$ has been defined in Eq. (60) and does not depend on $\boldsymbol{\alpha}$ if the fungal species are identical, as in this case $\operatorname{Var}(\boldsymbol{\alpha})=0$. Similarly, the biomass of species 1 when the plant is associating with a combination of a good (species 1) and a less beneficial mutualist (species 2) can be expressed as

$$
m_{\left(\alpha+\epsilon, p^{*}(\alpha, \alpha+\epsilon)\right)}^{*}=\frac{p^{*}(\alpha, \alpha+\epsilon)}{\mu_{m}}\left(\frac{q_{h m}(\alpha+\epsilon)\left(P^{*}(\epsilon)-1\right)-q_{h m} \frac{\epsilon}{2} P^{*}(\epsilon)}{d+p^{*}(\alpha, \alpha+\epsilon)}\right) .
$$

where $P^{*}(\epsilon)$ is given in Eq. (63). As $P^{*}(\epsilon)<P^{*}$, and $p^{*}(\alpha, \alpha+\epsilon)<p^{*}(\alpha+\epsilon, \alpha+\epsilon)$ (see Eq. 68), one can directly say that

$$
m_{\left(\alpha+\epsilon, p^{*}(\alpha, \alpha+\epsilon)\right)}^{*}<m_{\left(\alpha+\epsilon, p^{*}(\alpha+\epsilon, \alpha+\epsilon)\right)}^{*},
$$

i.e., the fungal biomass of a good mutualist is decreased by the presence of a less beneficial mutualist.

Let us first consider the fungal biomass of the less beneficial species (species 2) when the plant is associating with two individuals of species 2 :

$$
m_{\left(\alpha, p^{*}(\alpha, \alpha)\right)}^{*}=\frac{p^{*}(\alpha, \alpha)}{\mu_{m}}\left(\frac{q_{h m} \alpha\left(P^{*}-1\right)}{d+p^{*}(\alpha, \alpha)}\right) .
$$

The fungal biomass of species 2 when the plant is associating with a combination of a good (species 1) and a less beneficial mutualist (species 2) is given by

$$
m_{\left(\alpha, p^{*}(\alpha, \alpha+\epsilon)\right)}^{*}=\frac{p^{*}(\alpha, \alpha+\epsilon)}{\mu_{m}}\left(\frac{q_{h m} \alpha\left(P^{*}(\epsilon)-1\right)+q_{h m} \frac{\epsilon}{2} P^{*}(\epsilon)}{d+p^{*}(\alpha, \alpha+\epsilon)}\right) .
$$

I want to show that

$$
m_{\left(\alpha, p^{*}(\alpha, \alpha)\right)}^{*}<m_{\left(\alpha, p^{*}(\alpha, \alpha+\epsilon)\right)}^{*},
$$

i.e., the fungal biomass of the less beneficial mutualist is increased by the presence of a good mutualist. We know that $P^{*}(\epsilon) \rightarrow P_{\infty}^{*}$ for $\epsilon$ large enough (Eq. (65)). Hence the value of $P^{*}(\epsilon)$ 
has a lower bound, however $p^{*}(\alpha, \alpha+\epsilon)$ increases with increasing $\epsilon$. We can conclude that Eq. (75) holds true for $\epsilon$ large enough. If $\epsilon$ is small, $P^{*}(\epsilon)$ can be expressed as

$$
P^{*}(\epsilon)=P^{*}(0)-\frac{Q}{2 \alpha^{2} \sqrt{(1+Q)^{2}-4 Q\left(1+\frac{\eta_{P} \eta_{M}}{2}\right)}} \epsilon^{2}+\mathcal{O}(\epsilon),
$$

where $P^{*}(0)=P^{*}$. Hence, for $\epsilon$ small, Eq. (74) can be rewritten as

$$
\begin{aligned}
m_{\left(\alpha, p^{*}(\alpha, \alpha+\epsilon)\right)}^{*} & \frac{p^{*}(\alpha, \alpha+\epsilon)}{\mu_{m}}\left(\frac{q_{h m} \alpha\left(P^{*}-1\right)}{d+p^{*}(\alpha, \alpha+\epsilon)}\right) \\
& +\frac{p^{*}(\alpha, \alpha+\epsilon)}{\mu_{m}\left(d+p^{*}(\alpha, \alpha+\epsilon)\right)}\left(-\frac{Q}{2 \alpha^{2} \sqrt{(1+Q)^{2}-4 Q\left(1+\frac{\eta_{P} \eta_{M}}{2}\right)}} \epsilon^{2}+q_{h m} \frac{\epsilon}{2} P^{*}(\epsilon)\right) .
\end{aligned}
$$

Because $p^{*}(\alpha, \alpha)<p^{*}(\alpha, \alpha+\epsilon)$ (see Eq. (67)), we can conclude that also for $\epsilon$ small enough it holds

$$
m_{\left(\alpha, p^{*}(\alpha, \alpha)\right)}^{*}<m_{\left(\alpha, p^{*}(\alpha, \alpha+\epsilon)\right)}^{*},
$$

i.e., fungal biomass of a less beneficial fungus increases in the presence of a more beneficial species. Hence, in sum, the biomass of a fungus decreases in the presence of a less beneficial mutualist (Eq. (78)), and increases in the presence of a more beneficial mutualist (Eq. (72)). 